\title{
Comprehensive gene expression meta- analysis identifies signature genes that distinguish microglia from peripheral monocytes/macrophages in health and glioma
}

Verena Haage ${ }^{1 \dagger}$, Marcus Semtner ${ }^{1 \dagger}$, Ramon Oliveira Vidal ${ }^{1 \dagger}$, Daniel Perez Hernandez ${ }^{1}$, Winnie W. Pong ${ }^{2}$, Zhihong Chen ${ }^{3}$, Dolores Hambardzumyan ${ }^{3}$, Vincent Magrini ${ }^{4}$, Amy Ly ${ }^{4}$, Jason Walker ${ }^{4}$, Elaine Mardis ${ }^{4}$, Philipp Mertins ${ }^{1}$, Sascha Sauer ${ }^{1 \dagger}$, Helmut Kettenmann ${ }^{1+}$ and David H. Gutmann ${ }^{1,2^{*}+}$

\begin{abstract}
Monocytes/macrophages have begun to emerge as key cellular modulators of brain homeostasis and central nervous system (CNS) disease. In the healthy brain, resident microglia are the predominant macrophage cell population; however, under conditions of blood-brain barrier leakage, peripheral monocytes/macrophages can infiltrate the brain and participate in CNS disease pathogenesis. Distinguishing these two populations is often challenging, owing to a paucity of universally accepted and reliable markers. To identify discriminatory marker sets for microglia and peripheral monocytes/macrophages, we employed a large meta-analytic approach using five published murine transcriptional datasets. Following hierarchical clustering, we filtered the top differentially expressed genes (DEGs) through a brain cell type-specific sequencing database, which led to the identification of eight microglia and eight peripheral monocyte/macrophage markers. We then validated their differential expression, leveraging a published single cell RNA sequencing dataset and quantitative RT-PCR using freshly isolated microglia and peripheral monocytes/macrophages from two different mouse strains. We further verified the translation of these DEGs at the protein level. As top microglia DEGs, we identified P2ry12, Tmem119, S/c2a5 and Fcrls, whereas Emilin2, Gda, Hp and Sell emerged as the best DEGs for identifying peripheral monocytes/ macrophages. Lastly, we evaluated their utility in discriminating monocyte/macrophage populations in the setting of brain pathology (glioma), and found that these DEG sets distinguished glioma-associated microglia from macrophages in both RCAS and GL261 mouse models of glioblastoma. Taken together, this unbiased bioinformatic approach facilitated the discovery of a robust set of microglia and peripheral monocyte/macrophage expression markers to discriminate these monocyte populations in both health and disease.
\end{abstract}

Keywords: Microglia, Monocytes, Glioma, CNS; RNA sequencing, Microarray, Macrophages

\footnotetext{
* Correspondence: gutmannd@wustl.edu; David.Gutmann@mdc-berlin.de

†Verena Haage, Marcus Semtner and Ramon Oliveira Vidal; equal contribution as first authors.

${ }^{\dagger}$ Sascha Sauer, Helmut Kettenmann and David H. Gutmann; equal contribution as senior authors.

${ }^{1}$ Max Delbrück Center for Molecular Medicine in the Helmholtz Association, Berlin, Germany

${ }^{2}$ Department of Neurology, Washington University School of Medicine, Box

8111, 660 S. Euclid Avenue, St. Louis, MO 63110, USA

Full list of author information is available at the end of the article
}

(c) The Author(s). 2019 Open Access This article is distributed under the terms of the Creative Commons Attribution 4.0 International License (http://creativecommons.org/licenses/by/4.0/), which permits unrestricted use, distribution, and reproduction in any medium, provided you give appropriate credit to the original author(s) and the source, provide a link to the Creative Commons license, and indicate if changes were made. The Creative Commons Public Domain Dedication waiver (http://creativecommons.org/publicdomain/zero/1.0/) applies to the data made available in this article, unless otherwise stated. 


\section{Introduction}

Microglia represent the major population of myeloid cells (monocytes) in the healthy brain parenchyma, where they perform vital functions, ranging from homeostatic surveillance to serving as the first line of immune defense [45]. Microglia originate from primitive macrophages that exit the yolk sac at mouse embryonic day 8.5 , and subsequently colonize the neuroepithelium to become the resident CNS macrophage population [34]. Under certain pathological conditions, peripheral monocytes can enter the CNS from the blood through a disrupted blood brain barrier [13]. While there is little turnover of microglia in the healthy brain, blood monocytes/macrophages exhibit a high turnover rate [46]. In addition to their different origins, microglia and peripheral monocytes/macrophages have distinct functions in the setting of brain pathology. For example, opposing effects of microglia and infiltrated monocytes/macrophages have been reported in malignant brain tumors (glioblastoma) [5, 6, 9].

Defining the individual contributions of microglia and infiltrated monocytes/macrophages has been hampered by a lack of reliable markers that discriminate these two macrophage populations. First, while monocytes/macrophages are of haematopoetic origin, their transcriptome substantially overlaps with microglial gene expression $[7,16]$. Second, some of the genes/proteins used to distinguish these two populations are not exclusively expressed by either microglia or macrophages, but are only relatively enriched. This includes the protein tyrosine phosphatase receptor type $\mathrm{C}$ (CD45), the fractalkine receptor (CX3CR1), and the $\mathrm{C}-\mathrm{C}$ chemokine receptor type 2 (CCR2) [1, 4, 10, 15, 17, 25, 47]. Third, discriminatory genes frequently employed to identify peripheral monocytes/macrophages, such as CD45 or CCR2, can be induced in microglia associated with brain tumors (glioma). Similarly, blood-derived macrophages have been reported to decrease their $\mathrm{Ccr} 2$ expression upon entry into the brain under pathological conditions, while these same conditions induce $\mathrm{Ccr} 2$ expression in microglia [1, 4, 11, 32, 40, 47]. Lastly, while other monocyte population-specific markers have been identified, including TMEM119, it is not clear that they can reliably distinguish microglia from peripheral monocytes/macrophages in the normal brain and in the setting of CNS pathology $[3,5,7,14,28]$.

In an effort to generate a resource for discriminating microglia from peripheral monocyte/macrophage markers in the normal brain and in the setting of disease, we employed a meta-analytic approach using five published mouse transcriptomal datasets, where profiles from both microglia and peripheral monocyte/macrophage populations were included. In combination with several secondary selection filters and proteomic validation, a robust set of microglia and monocyte/macrophage DEGs was identified and shown to discriminate microglia from monocyte/ macrophages both in the normal brain and in the context of experimental murine glioma.

\section{Materials and methods}

Animals and ethics statement

All mice used for quantitative RT-PCR or proteomics validation were males, which were maintained on a C57BL/6J genetic background. Animals were handled according to governmental (LaGeSo) and internal (Max Delbrück Center for Molecular Medicine) rules and regulations. For quantitative RT-PCR validation, $C \times 3 c r 1^{\mathrm{EGFP} / \mathrm{WT}} ; C c r 2^{\mathrm{RFP} / \mathrm{WT}}$ mice were used to isolate microglia and peripheral monocyte/macrophages, respectively. Mice were kept in the animal facility using $12 \mathrm{~h}$ of light and dark cycle, with food and water ad libitum. All experiments were performed in strict accordance with the German Animal Protection Law as approved by the Regional Office for Health and Social Services in Berlin (Landesamt für Gesundheit und Soziales, Berlin, Germany, Permit Number (T0014/08, O360/09, A-0376/17). Adult mice were euthanized by intraperitoneal injection of pentobarbital (Narcoren, Merial GmbH, Hallbergmoos, Germany). All efforts were made to minimize pain and suffering.

Animals for the experimental glioma studies were housed in the Cleveland Clinic Biological Resource Unit or the Emory University Division of Animal Resources. All experimental procedures were approved by the Institutional Animal Care and Use Committee of the Cleveland Clinic (Animal Protocol 2013-1029; approved June 25, 2013) and Emory University (Protocol \#2003253; approved September 15, 2015), and performed in strict accordance with the recommendations in the Guide for the Care and Use of Laboratory Animals of the National Institutes of Health. All surgeries were performed under anesthesia, and all efforts were made to minimize suffering.

\section{Experimental high-grade glioma mouse models} Ntv-a;Ink4a-Arf-/-;Gli-luc mice developed gliomas following intracranial RCAS-PDGFB injection by $6-8$ weeks of age. Tumors were subsequently collected at 10-13 weeks of age [20]. Mice of both sexes were used in these experiments. Control mice were matched by genotype, gender, and age and did not receive RCAS injections.

\section{Fluorescence activated cell sorting (FACS) of microglia and spleen monocytes/macrophages}

12-14-week-old male C57/BL6 mice were transcardially perfused under deep anesthesia with 1x Phosphate Buffered Saline (PBS). Brains were isolated, and after removal of the cerebellum and brainstem, dissociated into a single-cell suspension using Adult Brain Dissociation 
Kit (Miltenyi, Bergisch Gladbach, Germany) and the gentleMACS dissociator (Miltenyi), according to manufacturer instructions. Subsequently, cells were washed in PBS, passed through a $35 \mu \mathrm{m}$ nylon mesh, counted and stained with anti-Mouse CD11b + PE-Cyanine7 (Life technologies | Thermo Fisher Scientific, Waltham, Massachusetts, USA) and anti-Mouse CD45 eFluor 450 (Life technologies | Thermo Fisher Scientific) for 20mins on ice. Spleens were manually dissociated in dissociation buffer (PBS containing 5.6\% Glucose and $15 \mathrm{mM}$ Hepes), and filtered through a $70 \mu \mathrm{m}$ strainer and then passed through a $35 \mu \mathrm{m}$ nylon mesh. Subsequently, the resulting single cell solution was centrifuged at $500 \mathrm{~g}$ for $5 \mathrm{~min}$, and red blood cells were lysed for $10 \mathrm{~min}$ in ACK buffer at room temperature. PBS was added, samples centrifuged, and the supernatant was discarded prior to staining the cells with anti-Mouse CD11b ${ }^{+}$PE-Cyanine7 (Life technologies) and anti-Mouse CD45 eFluor 450 (Life technologies), anti-Mouse Ly6G-FITC (eBioscience | Thermo Fisher Scientific, Waltham, Massachusetts, USA) and anti-Mouse Ly6C-PerCP/Cy5.5 (eBioscience | Thermo Fisher Scientific) for 20mins on ice. After staining, cells were washed once in PBS, and sorted on a FACS Aria flow cytometer (BD Biosciences, Franklin Lakes, USA) according to the specified gating strategy: microglia were sorted as $\mathrm{CD} 11 \mathrm{~b}^{+} \mathrm{CD} 45^{\text {low }}$ cells, whereas spleen monocytes/macrophages were isolated as $\mathrm{CD} 11 \mathrm{~b}^{+} \mathrm{CD} 45^{\text {high }}{ }^{\mathrm{Ly}} 6 \mathrm{G}^{\text {low }} \mathrm{Ly} 6 \mathrm{C}^{\text {high }}$ cells. For brain and spleen samples derived from $C x 3 c r 1^{\mathrm{GFP} / \mathrm{WT}} ; \mathrm{Ccr} 2^{\mathrm{RFP} / \mathrm{WT}}$ mice, cells were collected after centrifugation, washed in PBS, and the cell pellets snap frozen for storage at $-80^{\circ} \mathrm{C}$.

\section{Fluorescence activated cell sorting (FACS) of glioma- associated microglia and monocytes/macrophages from RCAS tumor mice}

Whole brains were collected from anesthetized and Ringer's solution-perfused 3-month-old Ntv-a;Ink4a-Arf ${ }^{1-}$;Gli-luc female and male mice and stored overnight in cold media. Tumors $(n=4)$, as well as age and gender matched forebrains from naïve animals $(n=4)$, were dissected and dissociated. Microglia and monocytes/macrophages were isolated using a Percoll density gradient for antibody-mediated flow sorting [8]. Forward Scatter (FSC) and Side Scatter (SSC) were used to determine viable cells, and appropriate controls were included for compensation and gating of stained populations (single, isotype and fluorescence minus one (FMO) controls) [2]. Two cell populations were collected from tumors: CD45 $5^{\text {high }}$ (infiltrated monocytes/macrophages); $\mathrm{CD} 45^{\text {low }}$ (microglia) cells that were also $\mathrm{CD}_{11 b^{+}}, \mathrm{F}_{11} \mathrm{r}^{+}, \mathrm{Ly}_{6 G^{\text {neg }}}, \mathrm{Sell}^{\text {neg }}, \mathrm{CD} 3^{\text {neg }}$, $\mathrm{CD} 19^{\text {neg }}$, and NK1.1 ${ }^{\text {neg }}$ cells. One population was collected from normal brain: $\mathrm{CD} 45^{\text {low }}$ that was also $\mathrm{CD} 11 \mathrm{~b}^{+}, \mathrm{F} 11 \mathrm{r}^{+}$, $\mathrm{Ly}_{6} \mathrm{G}^{\text {neg }}, \mathrm{Sell}^{\text {neg }}, \mathrm{CD}^{\text {neg }}, \mathrm{CD} 19^{\text {neg }}$, and $\mathrm{NK} 1.1^{\text {neg }}$. FACS samples were sorted directly into TRIzol (Life Technologies Corporation, Carlsbad, CA) for total RNA extraction.

\section{Gene expression analysis}

Gene expression datasets were identified by specifically choosing only studies that performed gene expression analysis of both microglia and peripheral monocyte/ macrophage populations at the same time, in order to minimize variation across sample preparation and analysis between laboratories. Datasets used for the meta-analysis included GSE46686 [33], GSE46690 [33], SRX424925 [22], GSE48579 [7], and GSE86573 [5] (Table 1).

\section{Microarray analysis}

Raw data files were downloaded, and analyzed using $\mathrm{R}$ package limma. The raw data was first normalized (RMA normalization), and the two groups were contrasted ( $\operatorname{lmFit}$ and eBayes functions) in order to obtain the fold changes and adjusted $p$-values between microglia and monocyte/ macrophage samples. The gene lists were further filtered for significant differential expression between monocytes/ macrophages and microglia using a fold change $(\log 2)$ cutoff of 2 and an adjusted p-value cutoff of 0.01. Each microarray dataset was individually analyzed.

\section{RNA sequencing analysis}

Processed data files were downloaded and analyzed using different approaches depending on the dataset. For normalized expression (fpkm) datasets, the values were first $\log$ transformed, and the fold changes calculated using limma ( $\mathrm{m} F i$ and eBayes function). For read counts datasets, the fold changes were calculated by DESeq2 package using default values. The gene lists were further filtered for significant differentially expressed genes between monocytes/macrophages and microglia using a fold change $(\log 2)$ cutoff of 2 and adjusted $p$-value cutoff of 0.01 . Each RNA sequencing dataset was individually analyzed.

\section{Meta-analysis of mouse RNA sequencing and microarray data} In order to compare all datasets, gene IDs were converted to gene symbols. Genes with increased expression in microglia or monocytes/macrophages were compared using the $\mathrm{R}$ package GeneOverlap to identify overlaps and intersections. We only selected genes as potential markers for each cell type that intersected in all of the datasets. A heat map (heatmap.2 function) was then constructed using the fold-change values (data were scaled) for all of these markers, and adjusted according to hierarchical clustering.

Analysis of mouse single-cell RNA sequencing (scRNA-Seq) Single cell data from the Tabular Muris Consortium was retrieved and analyzed [42]. All cells were labeled with 
Table 1 Gene expression datasets used for the meta-analysis

\begin{tabular}{|c|c|c|c|c|c|c|}
\hline Cell Type & Dataset & Tissue & Species & Platform & Data Format & Reference \\
\hline microglia & $\begin{array}{l}\text { GSM1134004 } \\
\text { GSM1134006 } \\
\text { GSM1134009 } \\
\text { GSM1134010 } \\
\text { GSM1134012 } \\
\text { GSM1134015 } \\
\text { GSM1134055 } \\
\text { GSM1134056 } \\
\text { GSM1134057 }\end{array}$ & brain & mouse & $\begin{array}{l}\text { Aroma } \\
\text { Aroma } \\
\text { Aroma } \\
\text { Expression console } \\
\text { Expression console } \\
\text { Expression console } \\
\text { RNA-seq } \\
\text { RNA-seq } \\
\text { RNA-seq }\end{array}$ & $\begin{array}{l}\text { CEL / microarray } \\
\text { CEL / microarray } \\
\text { CEL / microarray } \\
\text { CEL / microarray } \\
\text { CEL / microarray } \\
\text { CEL / microarray }\end{array}$ & Pong et al. 2013 [33] \\
\hline $\begin{array}{l}\text { peripheral monocytes/ } \\
\text { macrophages }\end{array}$ & $\begin{array}{l}\text { GSM1134005 } \\
\text { GSM1134007 } \\
\text { GSM1134008 } \\
\text { GSM1134011 } \\
\text { GSM1134013 } \\
\text { GSM1134014 } \\
\text { GSM1134052 } \\
\text { GSM1134053 } \\
\text { GSM1134054 }\end{array}$ & Bone marrow & mouse & $\begin{array}{l}\text { Aroma } \\
\text { Aroma } \\
\text { Aroma } \\
\text { Expression console } \\
\text { Expression console } \\
\text { Expression console } \\
\text { RNA-seq } \\
\text { RNA-seq } \\
\text { RNA-seq }\end{array}$ & $\begin{array}{l}\text { CEL / microarray } \\
\text { CEL / microarray } \\
\text { CEL / microarray } \\
\text { CEL / microarray } \\
\text { CEL / microarray } \\
\text { CEL / microarray }\end{array}$ & Pong et al. 2013 [33] \\
\hline microglia & $\begin{array}{l}\text { SRX424861 } \\
\text { SRX424857 }\end{array}$ & brain & mouse & $\begin{array}{l}\text { RNA-seq } \\
\text { RNA-seq }\end{array}$ & & Hickman et al. 2013 [22] \\
\hline $\begin{array}{l}\text { peripheral monocytes/ } \\
\text { macrophages }\end{array}$ & $\begin{array}{l}\text { SRX424925 } \\
\text { SRX424919 } \\
\text { SRX424904 } \\
\text { SRX424890 } \\
\text { SRX424880 } \\
\text { SRX424879 } \\
\text { SRX424878 }\end{array}$ & peritoneum & mouse & $\begin{array}{l}\text { RNA-seq } \\
\text { RNA-seq } \\
\text { RNA-seq } \\
\text { RNA-seq } \\
\text { RNA-seq } \\
\text { RNA-seq } \\
\text { RNA-seq }\end{array}$ & & Hickman et al. 2013 [22] \\
\hline microglia & $\begin{array}{l}\text { GSM1181585 } \\
\text { GSM1181587 } \\
\text { GSM1181589 }\end{array}$ & brain & mouse & $\begin{array}{l}\text { Affymetrix } \\
\text { Affymetrix } \\
\text { Affymetrix }\end{array}$ & $\begin{array}{l}\text { CEL / microarray } \\
\text { CEL / microarray } \\
\text { CEL / microarray }\end{array}$ & Butovsky et al. 2014 [7] \\
\hline $\begin{array}{l}\text { peripheral monocytes/ } \\
\text { macrophages }\end{array}$ & $\begin{array}{l}\text { GSM1181579 } \\
\text { GSM1181581 } \\
\text { GSM1181583 }\end{array}$ & spleen & mouse & $\begin{array}{l}\text { Affymetrix } \\
\text { Affymetrix } \\
\text { Affymetrix }\end{array}$ & $\begin{array}{l}\text { CEL / microarray } \\
\text { CEL / microarray } \\
\text { CEL / microarray }\end{array}$ & Butovsky et al. 2014 [7] \\
\hline microglia & $\begin{array}{l}\text { GSM2590424 } \\
\text { GSM2590425 } \\
\text { GSM2590426 }\end{array}$ & brain & mouse & $\begin{array}{l}\text { RNA-seq } \\
\text { RNA-seq } \\
\text { RNA-seq }\end{array}$ & & (Bowman et al. 2016 [5] \\
\hline $\begin{array}{l}\text { peripheral monocytes/ } \\
\text { macrophages }\end{array}$ & $\begin{array}{l}\text { GSM2590427 } \\
\text { GSM2590428 } \\
\text { GSM2590429 } \\
\text { GSM2590430 } \\
\text { GSM2590431 }\end{array}$ & blood & mouse & $\begin{array}{l}\text { RNA-seq } \\
\text { RNA-seq } \\
\text { RNA-seq } \\
\text { RNA-seq } \\
\text { RNA-seq }\end{array}$ & & (Bowman et al. 2016 [5] \\
\hline
\end{tabular}

the tissue of origin (brain myeloid cells or marrow) and processed with Seurat software (v2.3). The two datasets were normalized, scaled (data were regressed based on ERCC spiked-in controls), and aligned together (CCA dimension alignment). We generated $t$-SNEs from the aligned CCA dimensions, and violin plots were created to depict the expression of each of the markers on individual cells from the two tissues.

\section{Analysis of mouse RNA sequencing datasets from glioma- associated microglia and glioma-associated monocytes/ macrophages}

RNA sequencing datasets from high-grade gliomaassociated microglia and monocytes/macrophages isolated from experimental RCAS or GL261 tumors were extracted from GSE86573 or directly from the published manuscript (Table 1) [5]. Log2 fold changes in glioma-associated microglia relative to monocytes/macrophages were calculated for all microglia signature (SGMic) and monocyte/macrophage signature (SGMac) genes, including conventionally used markers $(\mathrm{Cx} 3 \mathrm{cr}$ ), $C d 11 b, C d 45, C c r 2)$, and the data plotted accordingly. Additionally, $\log 2$ fold changes in glioma-associated microglia relative to healthy microglia were calculated for all microglia signature (SGMic) genes and the data plotted accordingly.

\section{Quantitative RT-PCR validation}

Total RNA was extracted from FACS-sorted acutely isolated monocytes using ReliaPrep ${ }^{\mathrm{mt}}$ RNA Miniprep System (Promega Corporation; Madison, Wisconsin, USA), and first strand cDNA synthesis was performed using the 
PrimeScript $^{\text {tim }}$ RT reagent Kit (Takara, Kusatsu, Shiga, Japan) according to the manufacturer's instructions. Quantitative real-time PCR reactions to amplify $1 \mathrm{ng}$ of total cDNA for the selected genes (Table 2) were performed in a 7500 Fast Real-Time thermocycler (Applied Biosystems, Carlsbad, USA) using the SYBR Select Master Mix (Applied Biosystems | Thermo Fisher Scientific, Waltham, Massachusetts, USA). CT values were normalized using hypoxanthine guanine phosphoribosyltransferase $(H p r t)$. To ensure the specificity of each PCR product, melting curves were analyzed. The delta/delta $\mathrm{C}_{\mathrm{T}}$-method was employed for analysis of relative expression.

\section{Protein extraction and mass spectrometry analysis}

For each of the four independent proteomic runs, primary monocyte populations from four different male C57BL/6J mice were pooled and pelleted in PBS. The samples were solubilized in Laemmli buffer (LB) and subjected to SDS-PAGE. The proteome was focused into one gel band and processed as previously published $[26,39]$, with the use of an automated HTS PAL system (CTC Analytics, Switzerland). Peptides were extracted, purified and stored on reversed-phase (C18) StageTips [35]. Following elution, the peptides were lyophilized and resuspended in 0.1\% Formic Acid / 3\% Acetonitrile, prior to separation in a nano EasyLC 1200 (Thermo Fisher Scientific) with a $0.1 \times 200 \mathrm{~mm}$ MonoCap C18 HighResolution Ultra column (GL Sciences, Japan) at a flow rate of $300 \mathrm{~nL} / \mathrm{min}$ and a gradient from 5 to $95 \%$ B (80\% Acenotrile, 0,1\% Formic Acid) in $360 \mathrm{~min}$. The UHPLC was coupled online to an Orbitrap Q
Exactive plus mass spectrometer (Thermo Fisher Scientific) for mass spectrometry analysis. The mass spectrometer was set to acquire full-scan MS spectra $(300-1700 \mathrm{~m} / \mathrm{z})$ at a resolution of 17.500 after accumulation to an automated gain control (AGC) target value of $1 \times 10^{6}$ and maximum injection time of 20 $\mathrm{ms}$, and was operated in a data-dependent acquisition mode, selecting the 10 most abundant ions for MS/ MS analysis, with dynamic exclusion enabled (20 s). Charge state screening was enabled, and unassigned charge states and single charged precursors excluded. Ions were isolated using a quadrupole mass filter with a $1.2 \mathrm{~m} / \mathrm{z}$ isolation window, with a maximum injection time of $60 \mathrm{~ms}$. HCD fragmentation was performed at a normalized collision energy (NCE) of 26. The recorded spectra were searched against a mouse database from Uniprot (January 2017) using the MaxQuant software package (Version 1.5.2.8) [12] (with fixed modifications set to carbamylation of cysteines and variable modifications set to methionine oxidation). Peptide tolerance was $20 \mathrm{ppm}$ and the minimum ratio for LFQ was set to 2 . The false-discovery rate was set to $1 \%$ on protein and peptide level. Statistical analysis of the data set was performed using R-statistical software package (version 3.4.1), Prodigy (v0.8.2) and Perseus software (version 1.6.0.7).

For the data analysis, proteins that were only identified by site or were potential contaminants were excluded. Only those proteins discovered in at least three biological replicates were used for column-wise analysis using a two-sample t-test and a Benjamini-Hodgbergbased $\mathrm{FDR}<0.05$.

Table 2 Quantitative RT-PCR primers

\begin{tabular}{|c|c|c|}
\hline Gene & Forward Primer & Reverse Primer \\
\hline$\overline{C 3}$ & TGCCCCTTACCCCTTCATTC & CTCCAGCCGTAGGACATTGG \\
\hline Emilin2 & GCAGCTTGTGGAACTGCATC & TCGGTTGCTTCTGAGGGTTC \\
\hline F10 & GGTGAGTGAACCTTGCCCC & TGGCACGTTCCCGGTTAATA \\
\hline F5 & CACCCGTGATACCTGCGAAT & TCAGTGCGTTTGGTGAAGGT \\
\hline Fcrls & CTTGTGAGGCTGAAAACGCC & GCCATTCACCAAACGCACTT \\
\hline Gda & GACAGCGGCAAAATAGTGTTTCT & AGGCCTGGCATGAAGAACTC \\
\hline Gpr34 & CCTGGTCTAGGGAGTTTTGGG & GAGCAAAGCCAGCTGTCAAC \\
\hline$H p$ & CACTTGGTTCGCTATCGCTG & TCCATAGAGCCACCGATGAT \\
\hline Hprt & GATTAGCGATGATGAACCAGGTT & ССTCCCATCTCCTTCATGACA \\
\hline Mki67 & TGGTCACCATCAAGCGGAG & AGGCAGCTGGATACGAATGT \\
\hline $\mathrm{O} / \mathrm{fm} / 3$ & GCCGACTAGCTGCCTTAGAG & CCTCCCTTTCAAGACGGTCC \\
\hline P2ry12 & GCACGGACACTITCCCGTAT & GCCTTGAGTGTTTCTGTAGGGTA \\
\hline P2ry13 & CCTCATCGCTITCGACAGGT & GAACATCAGGGACCAGACGG \\
\hline Sell & TCATGGTCACCGCATTCTCG & CTTCACGGGAGGACTTGACG \\
\hline Siglec-H & ATGTCAGCTGCCCTCATATCC & CCTGTACCACATCTGCCAGG \\
\hline S/c2a5 & ACAGCTGGCACTITGAGGAG & TTGCCAGAGCAAGGACCAAT \\
\hline Tmem119 & CGGTCCTTCACCCAGAGC & TCGCAAGTAGCAGCAGAGAC \\
\hline
\end{tabular}




\section{mRNA library preparation and RNA sequencing}

Total RNA from flow-sorted cells was isolated by TRIzol-chloroform extraction. RNA samples were resuspended in Ambion Nuclease-free water (Life Technologies), snap frozen, and stored at $-80^{\circ} \mathrm{C}$. Prior to RNA sequencing, RNA was treated with TURBO DNA-free kit (Invitrogen | Thermo Fisher Scientific, Waltham, Massachusetts, USA) and assessed using the Agilent Eukaryotic Total RNA 6000 and Quant-iT ${ }^{\mathrm{TM}}$ RNA assay kit on a Qubit ${ }^{\mathrm{Tm}}$ Fluorometer (Life Technologies). cDNA was synthesized using the Ovation ${ }^{\odot}$ RNA-Seq method, and the Illumina paired-end LT indexing protocol used to construct an Illumina library from $500 \mathrm{ng}$ cDNA [19, 30]. Libraries were sequenced on an Illumina HiSeq, and15-22Mbp per lane of 100 basepair paired-end reads generated. RNA-Seq paired-end reads were processed using the TopHat suite [44] with Cufflinks [36, 37]. A fold-change and significance $(<0.05$ False Discovery Rate, FDR) for every gene was generated using cuffdiff [43].

\section{Data and software availability}

The previously unpublished datasets from gliomaassociated microglia and macrophages using the RCAS model are now available on the NCBI Gene Expression Omnibus (GEO Accession Series GSE65868).

\section{Results and Discussion}

Meta-analysis of gene expression datasets from microglia and peripheral monocyte/macrophage populations

To identify a reliable set of markers that distinguishes microglia from peripheral monocytes/macrophages, we leveraged a series of published RNA sequencing and microarray datasets from adult mouse brain microglia and peripheral monocyte/macrophage populations isolated from mouse bone marrow, blood, spleen and peritoneum. We only included studies that performed gene expression analyses of both populations, in order to minimize variations in the processing of the different samples between laboratories and the RNA analysis platforms $[5,7,22,33]$. Isolation protocols for microglia varied among the studies; however, microglia were commonly isolated by fluorescence-activated cell sorting (FACS) using CD11b and CD45 expression. We incorporated datasets of monocyte/macrophage populations from different tissue origins, since there were few published studies that performed simultaneous sequencing of microglia and monocyte/macrophage populations. As such, the selected datasets included RNA sequencing and microarray data from brainstem microglia $\left(\mathrm{CD} 11 \mathrm{~b}^{+} \mathrm{CD} 45^{\text {low }} \mathrm{Ly}_{6 \mathrm{G}^{-}}\right)$and bone marrow-derived macrophages $\left(\mathrm{CD} 11 \mathrm{~b}^{+} \mathrm{CD} 115^{+} \mathrm{Ly}_{6 \mathrm{G}^{-}}\right)$isolated by fluorescence-activated cell sorting [33], RNA sequencing of microglia $\left(\mathrm{CD} 11 \mathrm{~b}^{+} \mathrm{CD} 45^{+}\right)$and peritoneal macrophages $\left(\mathrm{CD} 11 \mathrm{~b}^{+} \mathrm{CD} 45^{+} ;[22]\right)$, microarray data from microglia $\left(\mathrm{CD} 11 \mathrm{~b}^{+} \mathrm{CD} 45^{\text {low }}\right)$ and spleen monocytes $\left(\mathrm{CD} 11 \mathrm{~b}^{+} \mathrm{Ly}_{6 \mathrm{C}^{+}}\right.$; [7]), and RNA sequencing of

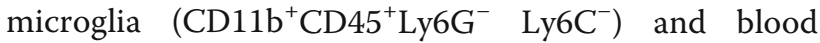
monocytes (CD11b $\left.{ }^{+} \mathrm{CD} 45^{+} \mathrm{Ly}_{6 G^{-}} \mathrm{Ly} 6 \mathrm{C}^{+}[5]\right)$.

Since the microarray and RNA sequencing data were analyzed using different methods and pipelines, we used the difference of gene expression (fold changes) between microglia and peripheral monocytes/macrophages within each dataset. Log-fold change values of enriched genes for each of the two populations were compared across the five different datasets (Fig. 1a). We identified 143 genes in microglia relative to peripheral monocytes/macrophages that were shared across the five analyzed studies. Next, hierarchical clustering was performed, revealing 13 microglia-enriched genes, including St3gal6 (Type 2 lactosamine alpha-2,3-sialyltransferase), P2ry13 (P2Y purinoceptor 13), P2ry12 (P2Y purinoceptor 12), Sparc (Secreted Protein Acidic And Cysteine Rich), Slco2b1 (Solute carrier organic anion transporter family member 2B1), Gpr34 (Probable G-protein coupled receptor 34), Slc2a5 (Solute carrier family 2, facilitated glucose transporter member 5), Sall1 (Sal-like protein 1), Siglec-H (Sialic acid-binding Ig-like lectin H), Olfml3 (Olfactomedin-like protein 3), Tmem119 (Transmembrane protein 119), Hpgds (Hematopoietic prostaglandin D synthase), and Fcrls (Fc receptor-like S, scavenger receptor) (Fig. 1b, left panel). For the monocyte/macrophage populations derived from bone marrow, blood, spleen or peritoneum, 145 significantly enriched and specific genes shared across all five datasets were identified. Following hierarchical clustering, two clusters were selected, representing 14 genes with the highest expression differences relative to microglia, including F10 (Coagulation factor X), Emilin2 (Elastin Microfibril Interfacer 2), F5 (Coagulation factor V), Slpi (Anti-leukoproteinase), Fn1 (Fibronectin), C3 (Complement C3), Anxa2 (Annexin A2), Gda (Guanine deaminase), Mki67 (proliferation marker protein Ki-67), Cd24a (CD24a antigen), S100a6 (S100 Calcium Binding Protein A6), Mgst1 (Microsomal glutathione S-transferase 1), Sell (L-selectin), and $H p$ (Haptoglobin) (Fig. 1b, right panel).

In order to evaluate the specificity of the identified markers for distinguishing microglia and monocytes/macrophages in the brain, we next assessed their expression in different CNS cell types using the Brain RNA-Seq transcriptome and splicing database [48]. Expression values were extracted for each gene within each marker set in microglia/macrophages, neurons, astrocytes, oligodendrocyte precursor cells, newly formed oligodendrocytes, myelinating oligodendrocytes and endothelial cells (Fig. 1c). For the set of microglia-enriched genes, we defined an expression threshold of 25 FPKM, such that any gene with a FPKM greater than 25 in any given CNS cell type other 
a

Microglia

Monocytes/Macrophages

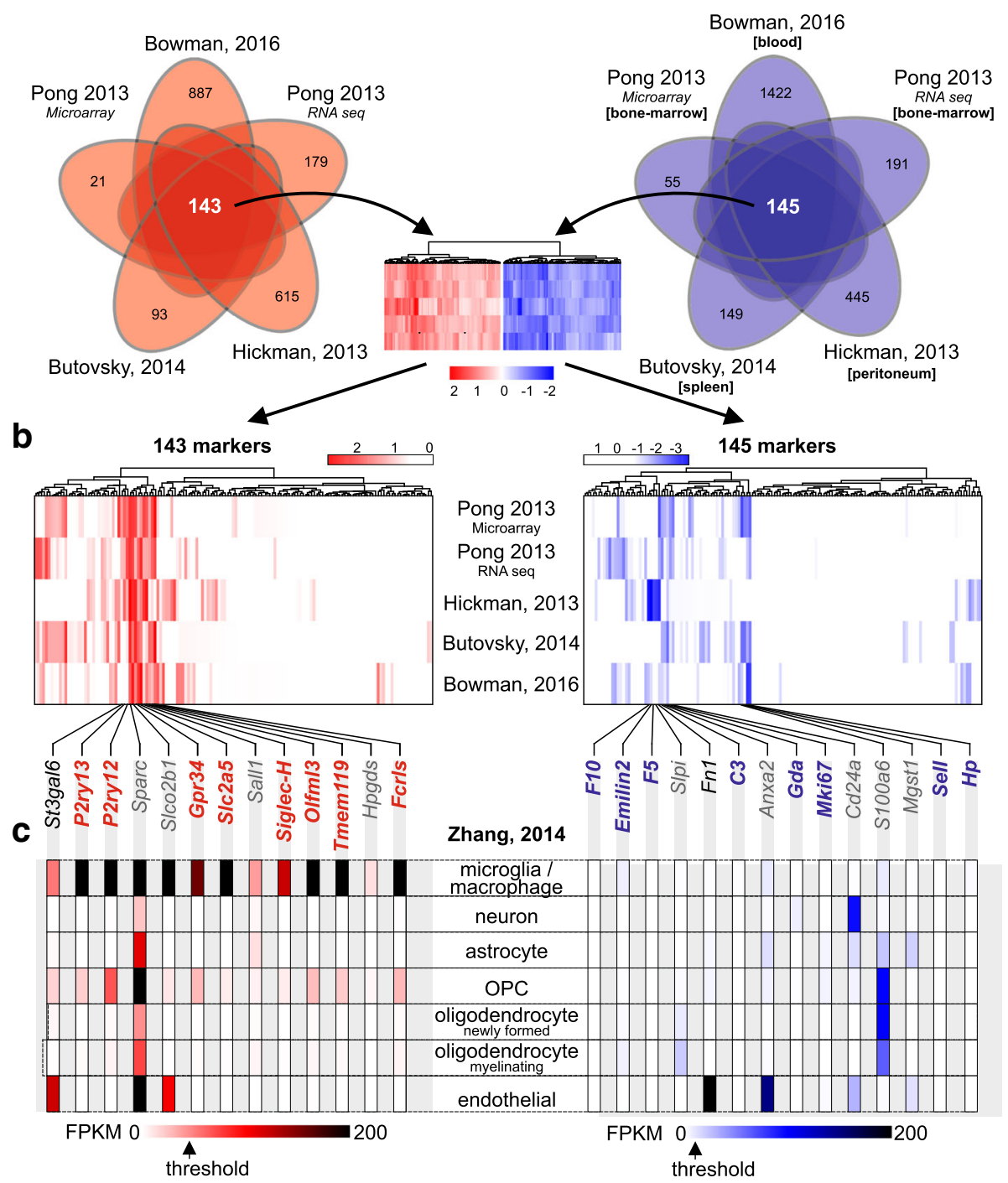

Fig. 1 Meta-analysis of mouse gene expression datasets from microglia and peripheral monocyte/macrophage populations derived from bone marrow, blood, spleen and peritoneum. (a) Venn diagram representing commonly expressed genes across the analyzed RNA sequencing and microarray datasets for microglia (red) and peripheral monocytes/macrophages isolated from bone marrow, blood, spleen or peritoneum (blue). Bioinformatic analysis of the five different expression studies identified 143 microglia-specific genes and 145 genes specific for peripheral monocytes/macrophages. The number of microglia and peripheral monocyte/macrophage genes exclusively expressed in each of the datasets is represented. (b) Heat map representing gene expression (Z-score) of the identified 143 microglia-specific and 145 peripheral monocyte/ macrophage-specific genes in each of the analyzed gene expression datasets. Hierarchical clustering identified modules of microglia-specific and of peripheral monocyte/macrophage-specific genes based on the dendrogram. For microglia, the module with the highest differential gene expression of identified microglia marker genes containing St3gal6, P2ry13, P2ry12, Sparc, Slco2b1, Gpr34, Slc2a5, Sall1, Siglec-H, Olfml3, Tmem119, Hpgds and Fcrls was selected for further analysis. For peripheral monocytes/macrophages, the two modules with highest differential gene expression containing the genes F10, Emilin2, F5, Slpi, Fn1, C3, Anxa2, Gda, Mki67, Cd24a, S100a6, Mgst1, Sell and Hp were selected for further analysis. (c) Representation of the expression levels for each of the selected microglia- and peripheral monocyte/macrophage-specific genes in different CNS cell types, including microglia/macrophages, neurons, astrocytes and oligodendrocyte precursor cells (OPCs), newly formed oligodendrocytes, myelinating oligodendrocytes and endothelial cells. FPKM values were extracted from the online database Brain-RNA-Seq (Zhang et al. 2014). The threshold FPKM expression value for excluding genes as microglia markers was set to 25, leading to elimination of St3gal6, Sparc, Slco2b1, Sall1 and Hpgds as microglia markers for further validation. Threshold FPKM expression value for excluding genes as peripheral monocyte/macrophage markers was set to 10, leading to elimination of Slpi, Fn1, Anxa2, Cd24a, S100a6 and Mgst1 as monocyte/ macrophage markers for further validation 
than microglia was excluded. As such, St3gal6 and Slco2b1 were eliminated from further analysis due to their high expression in endothelial cells, Sparc because of its high expression in all CNS cell types, and Sall1 and Hpgds due to their low expression in microglia/macrophages. The 14 identified monocyte/macrophage markers exhibited low expression levels in microglia [48], consistent with the notion that there are few, if any, peripheral monocytes/macrophages in the healthy brain. We defined 10 FPKM reads as a threshold for the exclusion of monocyte/macrophage markers due to their expression in other brain cell types. For this reason, we eliminated Slpi due to its expression in newly formed and myelinating oligodendrocytes, $F n 1$ and Anxa2 due to their high expression in endothelial cells, and $C d 24 a$ for its high expression in neurons and endothelial cells. S100a6 and Mgst1 were also excluded, since they were highly expressed in astrocytes, OPCs and oligodendrocytes, astrocytes and endothelial cells, respectively. Taken together, a panel of eight specific microglia signature genes (SGmic: P2ry13, P2ry12, Gpr34, Slc2a5, Siglec-H, Olfml3, Tmem119, Fcrls) and eight specific peripheral monocyte/macrophage signature genes (SGmac: F10, Emilin2, F5, C3, Gda, Mki67, Sell, Hp) were identified.

Since these analyses included monocyte/macrophage populations derived from blood, bone marrow, spleen, and peritoneum, we also analyzed the expression of the identified SGmac genes across the different populations as shown in Additional file 1: Figure S1. While all markers were expressed in the different monocyte subsets, $\mathrm{Hp}$, Sell and Gda were highly expressed in blood monocytes relative to spleen, bone marrow-derived or peritoneal macrophages. In addition, peritoneal macrophages exhibited high levels of Fn1, Slpi, Emilin2 and F10 expression, while Hp, Sell, Mgst1 and S100a6 were expressed at lower levels. Bone marrow-derived monocytes showed highest expression of $C d 24 a$ and Mki67, C3 and Fn1.

\section{Validation of SGmic and SGmac in single-cell sequencing datasets}

To provide a second method for assessing the utility of these monocyte marker sets in discriminating microglia from peripheral monocytes/macrophages, we leveraged a recently published study using single-cell sequencing of microglia and bone marrow-derived cells [42]. For myeloid brain cells, data were collected from 4762 cells, while for bone marrow cells, data were derived from 5353 single cells. The bone marrow-derived cells were next sorted in silico for $C d 11 b$ and $C d 45$ to identify monocytes; however, almost all of the cells expressed these two markers, making discrimination impossible. We next tried to sort for the fractalkine receptor (Cx3cr1), since peripheral monocytes/macrophages express only low levels of $C x 3 c r 1$ [17, 24]. Unfortunately,
Cx3cr1 was expressed in myeloid brain cells, precluding its use to presort monocytes/macrophages from bone marrow-derived cells in silico. Thus, we compared the expression of the two marker sets in the brain myeloid fraction (termed microglia; MG) with the bone marrow cells (termed BM). Expression of each of the eight identified signature genes for microglia (Fig. 2a) and peripheral monocytes/macrophages (Fig. 2b) was normalized, and independently represented for each of the two populations.

Of the eight SGmic genes, six (P2ry13, P2ry12, Gpr34, Slc2a5, Olfml3, Tmem119) were present in the single-cell sequencing data, and all were enriched in the microglia population relative to the bone marrowderived cells. P2ry12, Olfml3 and Tmem119 were enriched in nearly all of the sequenced cells, whereas P2ry13, Slc2a5 and Gpr34 were enriched, but not exclusively expressed in all of the sequenced microglia. Siglec-H and Fcrls were not present in the single cell RNA sequencing dataset. Conversely, all eight SGmac genes (F10, Emilin2, F5, C3, Gda, Mki67, Sell, Hp) were present in the bone marrow single cell sequencing dataset [42]: $\mathrm{Hp}$ and $\mathrm{C} 3$ were enriched in the bone marrow cells compared to microglia; however, C3 was also expressed in a small microglia population at elevated levels. All other monocyte/macrophage marker genes were enriched in bone marrow-derived cells relative to brain myeloid cells, with varying expression levels across the sequenced single cells. Mki67 and Gda were enriched at higher levels than Emilin2 and F5. Sell and F10 expression was evenly distributed across the sequenced cells, with $F 10$ showing comparatively lower expression levels. In addition, the t-SNE distribution of microglia and bone marrow cells was examined, and the expression of the eight identified signature genes plotted for each of the populations as shown in Additional file 2: Figure S2. The brain myeloid cells clustered homogenously in the middle of the plot, and the expression of the eight SGmic genes correlated with that cluster. In contrast, the bone marrow cells formed six different clusters distributed at the periphery of the microglia cluster, of which, all of the SGmac genes were localized to at least two of these clusters.

We also examined the expression of four canonical microglia/macrophage markers $(C d 11 b, C d 45, C x 3 c r 1$ and $C$ cr2) within the Tabula Muris dataset (Fig. 2c). While $C d 11 b$ and $C d 45$ were expressed in both myeloid brain cells and bone marrow-derived cells, Cx3cr1 was enriched in microglia. Expression of the commonly used peripheral monocyte/macrophage marker Ccr2 was only slightly enriched in the bone marrow cells, with very low levels of expression. Taken together, the classically used monocyte population markers underperformed as discriminatory genes relative to Tmem119, P2ry12, and Olfml3 as microglia 
a
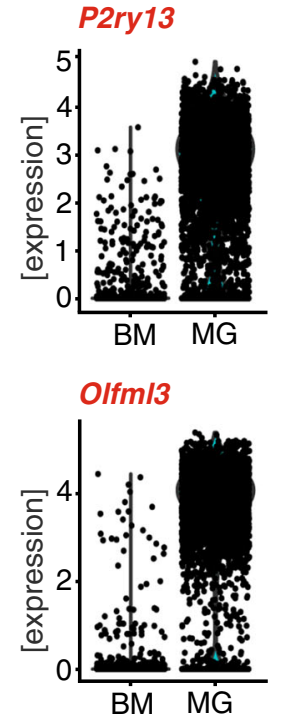

b
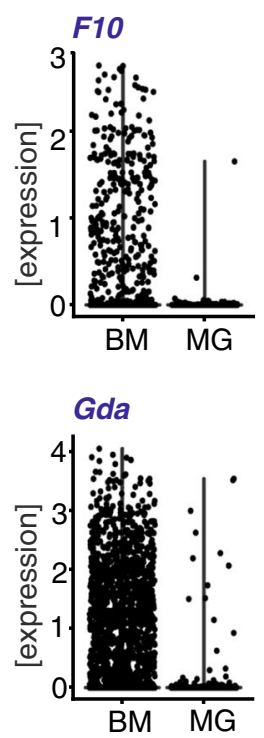

C

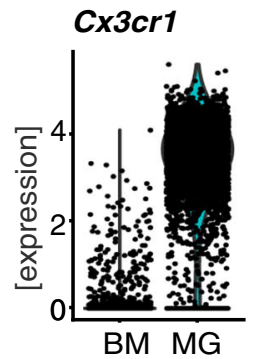

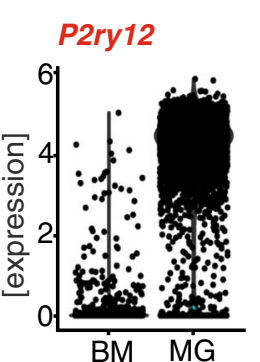
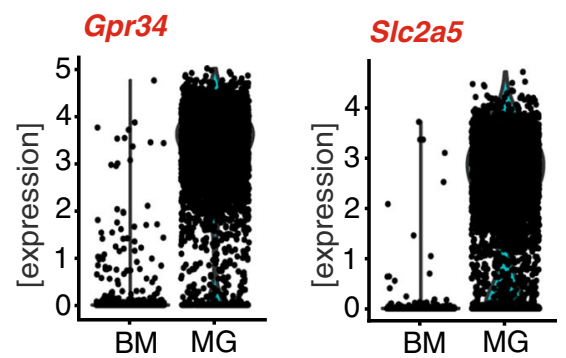

Tmem119

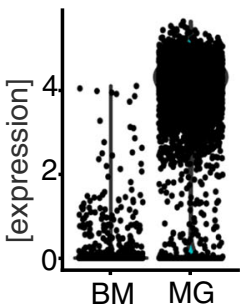

Emilin2
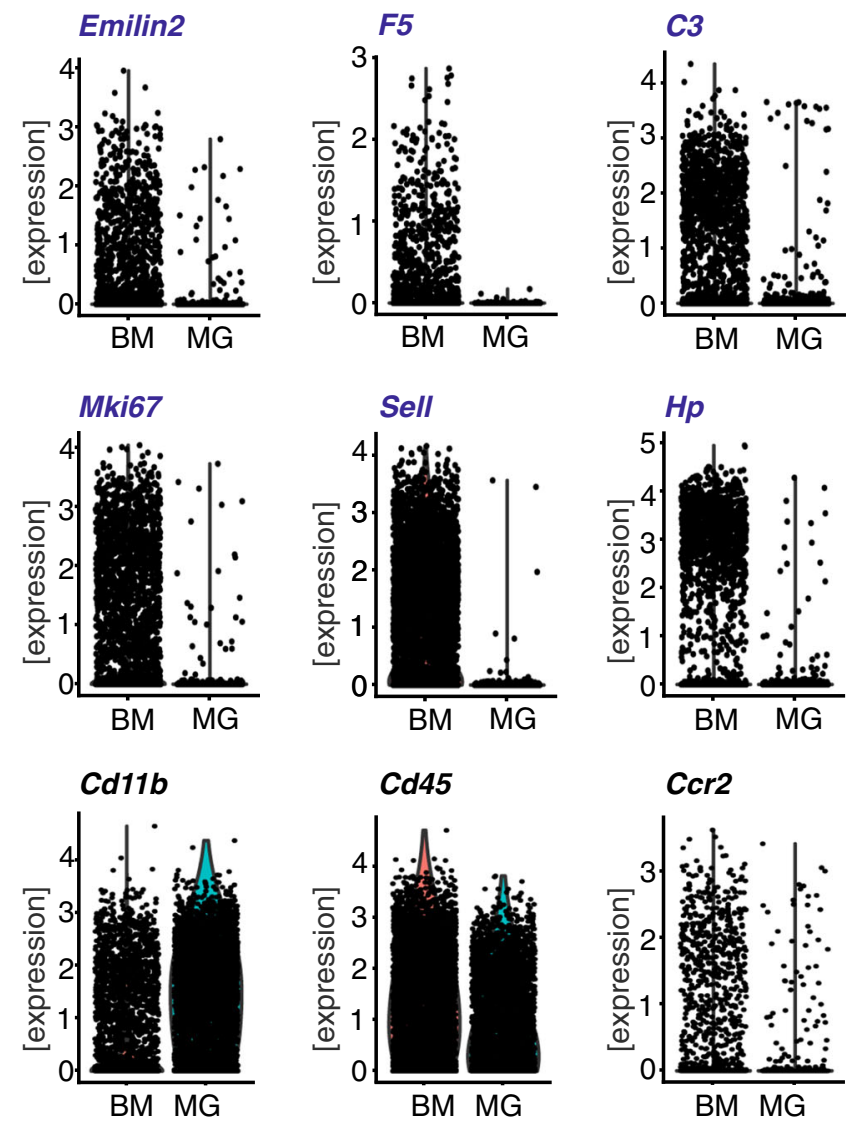

Fig. 2 Validation of SGmic and SGmac gene expression in single-cell RNA sequencing datasets of brain myeloid cells (microglia) and bone marrow cells. Graph depicts normalized expression of single cell sequencing data of brain myeloid cells (termed MG for microglia) and bone marrow cells (BM) extracted from the Tabular Muris dataset [42] for (a) SGmic and (b) SGmac genes relative to the conventional markers Cd11b, Cd45, Cx3cr1 and Ccr2 (c) 
specific markers and $\mathrm{Hp}, \mathrm{C} 3, \mathrm{Mki67,Gda}$ and Sell as monocyte/macrophage markers.

\section{SGmic and SGmac genes discriminate freshly isolated microglia from peripheral monocytes/macrophages}

To determine the discriminatory capabilities of these identified markers, we employed two different approaches. First, we isolated microglia as $\mathrm{CD} 11 \mathrm{~b}^{+} \mathrm{CD} 45^{\text {low }}$ cells and spleen monocytes/macrophages as $\mathrm{CD}_{11 b^{+} \mathrm{CD} 45^{\text {high }}{ }^{\mathrm{Ly}} 6 \mathrm{G}^{\text {low }} \text { Ly6C }}{ }^{\text {high }}$ from 12-week-old male C57BL/6J mice by fluorescence-activated cell sorting (FACS), and determined their relative expression by RT-qPCR analysis (Fig. 3a). All eight SGmic markers were enriched and exclusively expressed in microglia relative to spleen monocytes/macrophages: Olfml3, Fcrls, and Gpr34 exhibited the highest expression, with only P2ry12 demonstrating very low expression levels in spleen monocytes/macrophages as shown in Additional file 3: Figure S3a. In addition, all eight SGmac markers were enriched in spleen monocytes/macrophages relative to microglia: F10, Emilin2, C3, Gda and $H p$ were exclusively and highly expressed in spleen monocytes/macrophages, whereas F5, Mki67 and Sell were detected at low levels in microglia (Additional file 3: Figure S3a).

Using a second complementary method, we examined the SGmic and SGmac genes in $C \times 3 c r 1^{\mathrm{GFP} / \mathrm{WT}} ; \mathrm{Ccr} 2^{\mathrm{RFP} /}$ WT mice, where green fluorescent protein (GFP) expression is driven by the fractalkine receptor $(\mathrm{C} x 3 \mathrm{cr} 1)$ promoter, revealing microglia in the healthy brain as $\mathrm{GFP}^{+}$ cells. Conversely, red fluorescent protein (RFP) expression is controlled by the Ccr 2 promoter, allowing for the identification of peripheral monocytes/macrophages as $\mathrm{RFP}^{+}$cells. While this mouse model was originally designed to distinguish CNS resident microglia from peripheral monocytes/macrophages, several studies have identified low $C x 3 c r 1$ expression in the latter population $[17,24,25]$. Consistent with this observation, we detected a $\mathrm{RFP}^{+} \mathrm{GFP}^{+}$, as well as a $\mathrm{RFP}^{+} \mathrm{GFP}^{-}$, population in the $C x 3 c r 1^{\mathrm{GFP} / \mathrm{WT}} ; C c r 2^{\mathrm{RFP} / \mathrm{WT}}$ spleen samples. Using this strain, we isolated microglia $\left(\mathrm{GFP}^{+} \mathrm{RFP}^{-}\right.$cells) from the healthy brain and two populations of spleen monocytes/macrophages $\left(\mathrm{RFP}^{+} \mathrm{GFP}^{+}\right.$and $\mathrm{RFP}^{+} \mathrm{GFP}^{-}$cells) from 8 to 12 -week-old male mice. We defined the $\mathrm{RFP}^{+} \mathrm{GFP}^{+}$cells as the spleen monocyte/macrophage population (Fig. 3b).

Next, we determined the expression of the SGmic and SGmac marker sets across the three populations, focusing primarily on $\mathrm{GFP}^{+} \mathrm{RFP}^{-}$microglia and $\mathrm{RFP}^{+} \mathrm{GFP}^{+}$ spleen monocytes/macrophages. All eight SGmic genes were enriched in the $\mathrm{GFP}^{+} \mathrm{RFP}^{-}$microglia population relative to $\mathrm{RFP}^{+} \mathrm{GFP}^{+}$cells, as well as to $\mathrm{RFP}^{+} \mathrm{GFP}^{-}$cells (Fig. 3b; Additional file 3: Figure S3b). In these analyses, Tmem119, Fcrls, Olfml3 and Slc2a5 exhibited the highest levels of expression. As observed in C57BL/6J WT mice
(Fig. 3a), low levels of P2ry12 expression were detected in both spleen monocyte/macrophage populations (Additional file 3: Figure S3b). Conversely, the eight SGmac genes were enriched in the $\mathrm{RFP}^{+} \mathrm{GFP}^{+}$population relative to $\mathrm{GFP}^{+} \mathrm{RFP}^{-}$microglia, with Emilin2, Gda and $\mathrm{Hp}$ showing the highest expression levels. Sell was the only marker expressed at higher levels in the $\mathrm{RFP}^{+} \mathrm{GFP}^{-}$ population (Additional file 3: Figure S3b), but was still enriched in both isolated spleen monocyte/macrophage populations as compared to $\mathrm{GFP}^{+} \mathrm{RFP}^{-}$microglia.

\section{Proteomic analysis confirms P2ry12, Tmem119, Slc2a5, and Fcrls as microglia markers, and Gda, Hp, C3, Mki67 and Emilin2 as monocyte/macrophage markers}

To provide a third line of evidence for the discriminatory ability of the identified SGmic and SGmac gene sets, we sought to confirm their differential expression at the protein level. Proteomics data were generated from microglia $\left(\mathrm{CD} 11 \mathrm{~b}^{+} \mathrm{CD} 45^{\text {low }}\right)$ and spleen monocytes/macrophages $\quad\left(\mathrm{CD} 11 \mathrm{~b}^{+} \mathrm{CD} 45^{\text {high }} \mathrm{Ly}_{6 \mathrm{G}^{\text {low }}} \mathrm{Ly}_{6 \mathrm{C}^{\text {high }}}\right)$ isolated by FACS from 12-week-old naïve C57BL/6J male mice. Protein expression levels of the SGmic and SGmac genes were calculated from the measured IBAQ intensities and normalized to Gapdh (Fig. 4a), and the t-test differences in protein expression determined (Fig. 4b). Since P2ry13 and Gpr34 could not be identified in the proteomic analysis, they were excluded. Enrichment of P2ry12, Slc2a5, Olfml3, Tmem119, and Fcrls protein levels were observed in microglia relative to spleen monocytes/macrophages. When normalized to Gapdh, P2ry12 expression was highest in microglia, followed by Tmem119, Slc2a5 and Fcrls. Olfml3 protein expression, however, was higher in spleen monocytes/ macrophages than in microglia. The switch in quantification can be explained by the method of protein extraction for proteomic analysis. Since Olfml3 is a secreted protein, only the intracellular amount of the protein can be accurately recovered and quantified. When compared to spleen monocytes/macrophages, P2ry12 showed the highest t-test difference, followed by Tmem119, Slc2a5, Fcrls and Olfml3.

The monocyte/macrophage markers F5 and F10 could not be detected in the proteomics analysis, which might be explained by the fact that both are secreted proteins and intracellular protein levels might fall below proteomic detection levels. Normalized to Gapdh, Gda and Hp showed the highest protein expression levels in peripheral monocytes/macrophages, followed by C3, Mki67 and Sell. For Gda and $\mathrm{Hp}$, very low protein expression in microglia was observed. Relative to microglia, C3, Mki67, Gda and Hp protein levels showed the highest t-test difference, followed by Emilin2 and Sell. As a reference, protein expression of the conventionally used microglia/macrophage markers, Cx3cr1, Cd11b, Cd45 
a
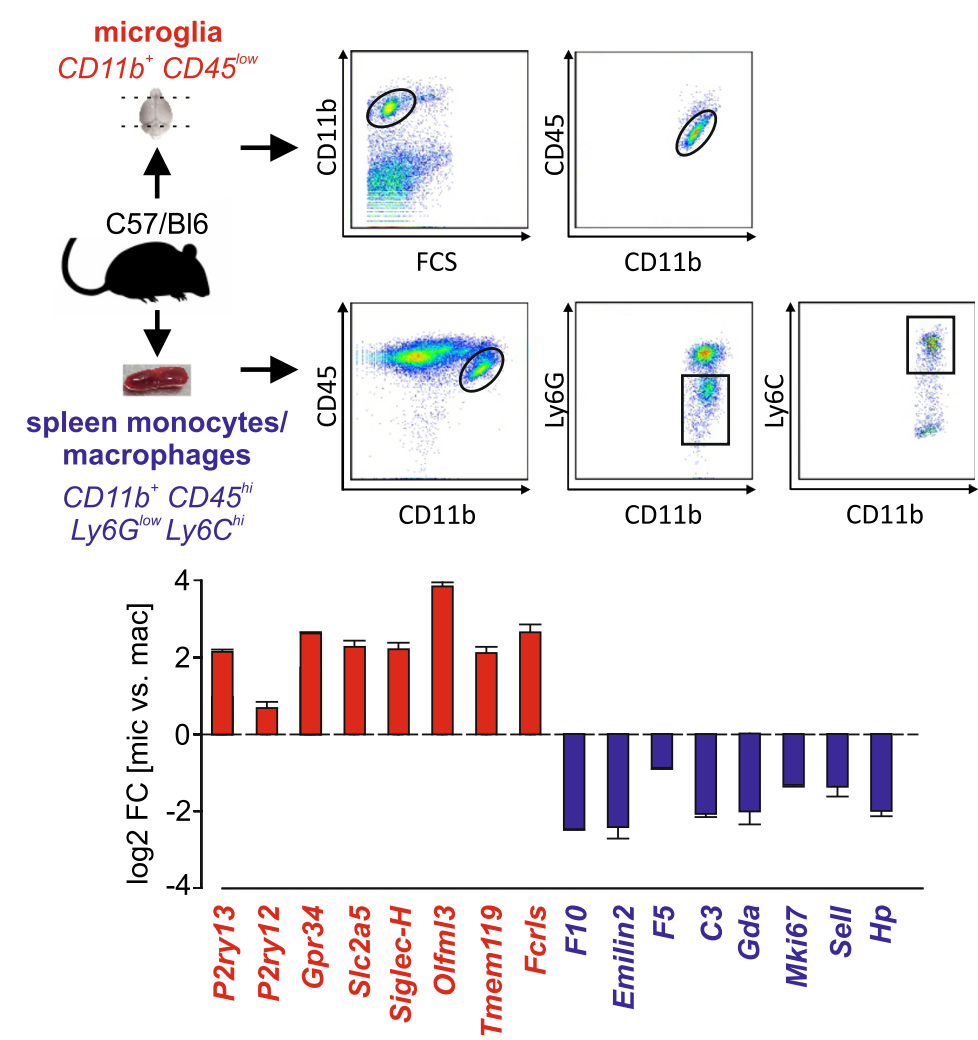

b
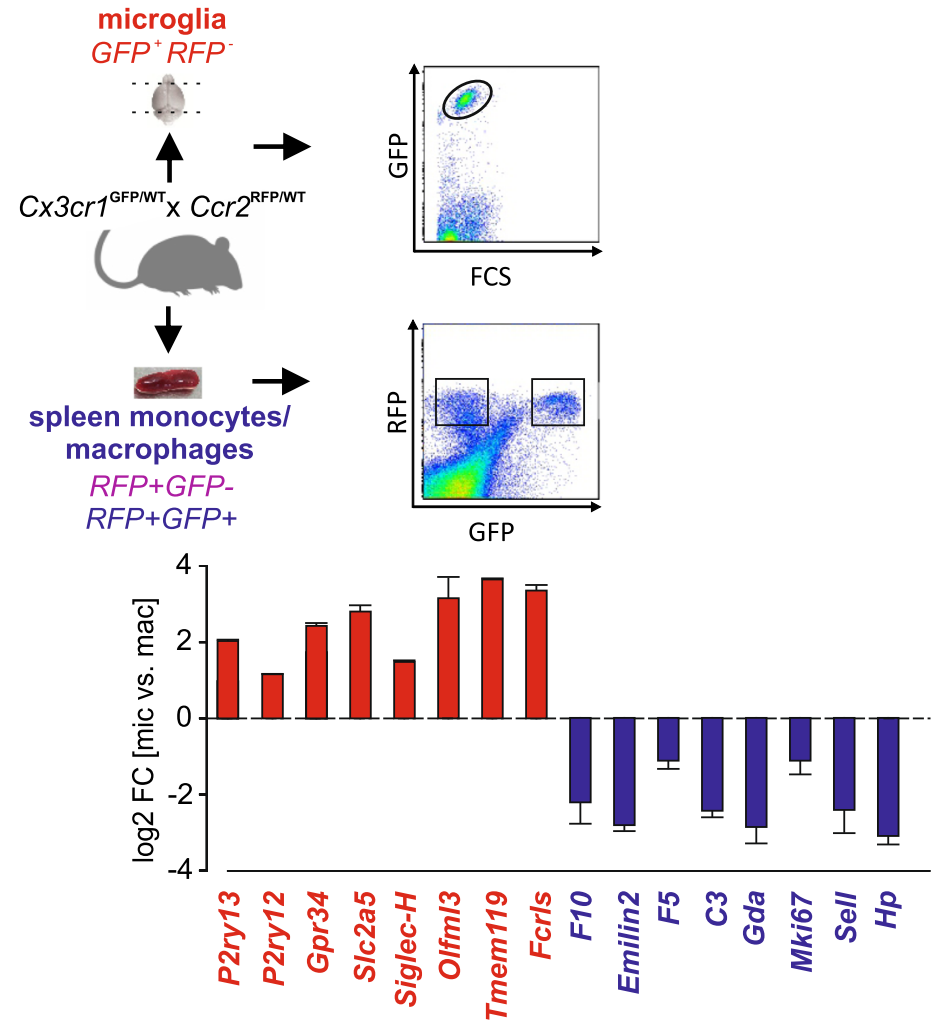

Fig. 3 (See legend on next page.) 
(See figure on previous page.)

Fig. 3 Validation of SGmic and SGmac genes by quantitative RT-PCR analysis in microglia and spleen monocytes/macrophages freshly isolated from two different mouse models. (a) Microglia and spleen monocytes/macrophages were freshly isolated from 12-weeks old male C57BL/6J WT mice by FACS. Microglia were first gated as CD11 b cells against forward scatter (FSC) and subsequently selected as CD45 ${ }^{\text {low }}$ expressing cells (microglia; red; $C D 11 b+C D 45^{\text {low }}$ ). Spleen monocytes/macrophages were first gated based on CD11 $b^{+}$and CD45 $5^{\text {high }}$ expression, followed by gating for Ly6G ${ }^{\text {low }}$ and Ly6C $C^{\text {high }}$ expression (spleen monocytes/macrophages; blue; CD11b + CD45 ${ }^{\text {low }}$ Ly6G ${ }^{\text {low }}$ Ly6C ${ }^{\text {high }}$ ). Expression of SGmic (P2ry13, P2ry12, Gpr34, Slc2a5, Siglec-H, Olfm/3, Tmem119, and Fcrls) and SGmac (F10, Emilin2, F5, C3, Gda, Mki67, Sell, Hp) genes was assessed in microglia (CD11 b $\mathrm{CD}^{+} 5^{\text {low }}$ ) and spleen monocytes/macrophages (CD11 b $\mathrm{CD}^{+} 5^{\text {high }}$ Ly6 $\mathrm{G}^{\text {low }}$ Ly6 $\mathrm{C}^{\text {high }}$ ) by quantitative RT-PCR. (b) Microglia and spleen monocytes were freshly isolated from 8 to 12 weeks old male $C \times 3 \mathrm{Cr}$ GFP $^{\mathrm{G} N T}$; CCr2 ${ }^{\mathrm{RFP} / \mathrm{NT}}$ mice by FACS. Microglia were gated as GFPexpressing cells against FSC (microglia; red; GFP ${ }^{+} \mathrm{RFP}^{-}$). Spleen monocytes/macrophages were isolated as RFP-expressing cells and sorted as two populations based on their GFP-expression levels as RFP+GFP ${ }^{+}$(spleen monocytes/macrophages; blue) and RFP GFP $^{-}$cells (spleen monocytes/ macrophages; purple). Expression of SGmic (P2ry13, P2ry12, Gpr34, Slc2a5, Siglec-H, Olfm/3, Tmem119, and Fcrls) and SGmac (F10, Emilin2, F5, C3, Gda, Mki67, Sell, Hp) genes was assessed in microglia (GFP RFP- cells) and spleen monocytes/macrophages (RFP ${ }^{+} \mathrm{GFP}^{+}$cells) by quantitative RT-PCR. Bar graphs represent the log fold change expression of each gene normalized to Hprt and in the isolated microglia population relative to the peripheral monocytes/macrophage population $\left(\mathrm{CD} 11 \mathrm{~b}^{+} \mathrm{CD}_{4} 5^{\text {high }}\right.$ Ly6G $^{\text {low }}$ Ly6C $\mathrm{C}^{\text {high }}$ or RFP $\mathrm{GFP}^{+}$cells; blue; $n=3$ )

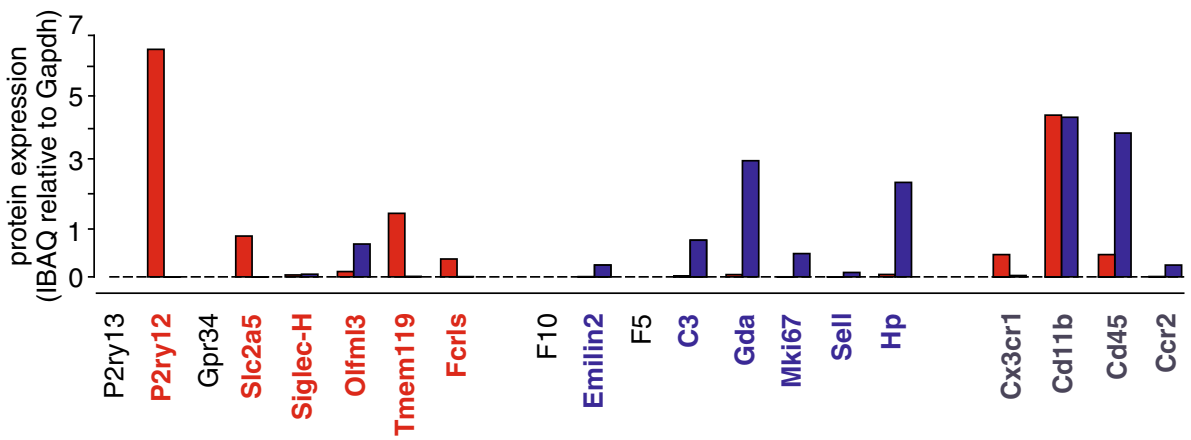

\section{b}
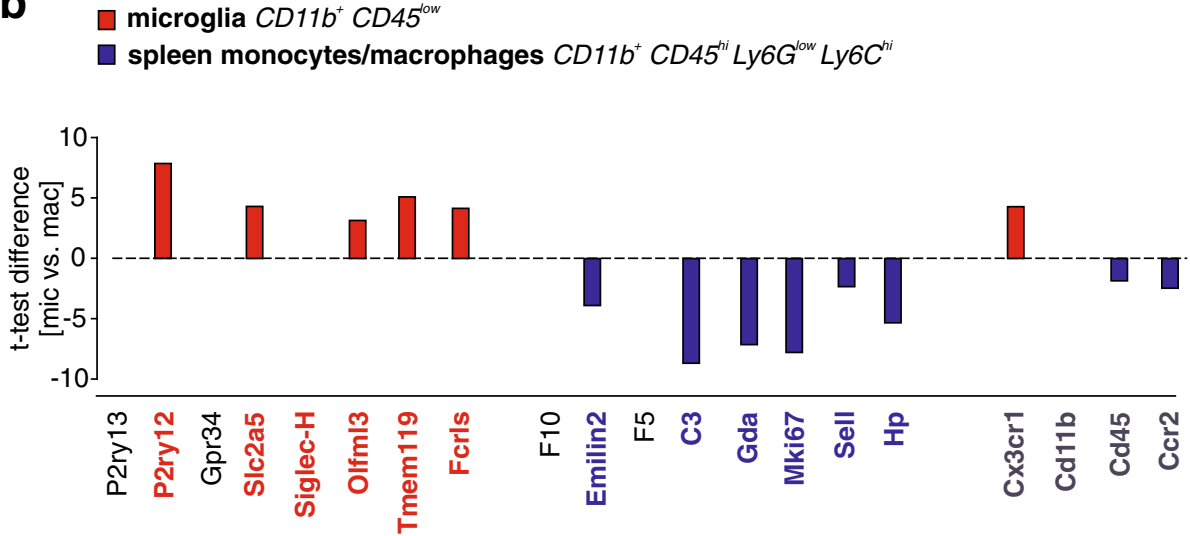

Fig. 4 Protein expression of SGmic and SGmac markers in freshly isolated microglia and spleen monocytes/macrophages. (a) Protein expression of SGmic and SGmac genes and reference markers (Cx3cr1, Cd11b, CD45, Ccr2) in microglia and spleen monocytes/macrophages. IBAQ intensities of each protein normalized to Gapdh intensity are shown. (b) Proteomic data were analyzed by a column-wise analysis using a two-sample t-test and a Benjamini-Hodgberg-based FDR $<0.05$. T-test difference of SGmic, SGmac and reference marker expression in microglia relative to spleen monocytes/macrophages is shown $(n=4)$ 
and Ccr2, were also analyzed. Cd11b protein was highly expressed in both microglia and spleen monocytes/macrophages, Cx3cr1 was enriched in microglia, and $\mathrm{Cd} 45$ and Ccr2 were mildly enriched in peripheral spleen monocytes/ macrophages when compared to microglia. Taken together, we provide the first transcriptomic and proteomic evidence for $\mathrm{Hp}, \mathrm{Gda}$, Sell, C3, Mki67 and Emilin2 as specific markers for peripheral monocytes/ macrophages and P2ry12, Tmem119, Slc2a5 and Fcrls as microglia-specific markers.

\section{SGmic and SGmac genes discriminate between glioma- associated microglia and monocytes/macrophages}

Using the SGmic and SGmac gene sets, we next explored their utility for discriminating between microglia and infiltrated monocytes/macrophages in the setting of brain cancer. For these studies, we employed datasets derived from two different experimental murine glioblastoma models, the induced RCAS-TVA system [20] and the GL261 glioma explant system [38].

First, we generated RCAS/TVA-induced tumors in Ntv-a;Ink4a-Arf ${ }^{-1}$;Gli-luc mice by RCAS-mediated expression of PDGFB, and subsequently isolated tumor-associated microglia and monocytes/macrophages based on $\mathrm{CD}_{11 b^{+}}, \mathrm{CD} 45^{\text {low, }} \mathrm{F}_{11 r^{+}}, \mathrm{Ly}_{6 \mathrm{G}}{ }^{\text {neg }}$, Sell $^{\text {neg }}$, $\mathrm{CD} 3^{\text {neg }}, \mathrm{CD} 19^{\text {neg }}$, and NK1.1 ${ }^{\text {neg }}$ (microglia) and CD11b ${ }^{+}$, $\mathrm{CD} 45^{\text {high }}, \mathrm{F} 1 \mathrm{r}^{+}, \mathrm{Ly}_{6 \mathrm{G}^{\text {neg }}}, \mathrm{Sell}^{\text {neg }}, \mathrm{CD} 3^{\text {neg }}, \mathrm{CD} 19^{\text {neg }}$, and NK1.1 ${ }^{\text {neg }}$ (monocytes/macrophages) gating. RNA sequencing was performed, and the $\log 2$ fold changes in expression were calculated for each gene (Fig. 5a). In the RCAS/TVA system, all SGmic genes (P2ry13, P2ry12, Gpr34, Slc2a5, Siglec-H, Olfml3, Tmem119, Fcrls) were enriched in glioma-associated microglia relative to glioma-associated monocytes/macrophages, with Slc2a5, Siglec-H, Gpr34 and P2ry12 showing the highest differential expression. Similarly, the SGmac markers F10 and $\mathrm{Hp}$ were increased in glioma-associated monocytes/macrophages ( $\log 2$-fold changes $=3-4.7$ ), whereas Emilin2, Gda and Sell were slightly increased (log2-fold changes =1.1-1.8) and C3 and Mki67 only barely enriched. F5 did not show any changes in gene expression between glioma-associated microglia and monocytes/macrophages. The canonical reference genes, $C \times 3 c r 1, C d 11 b$ and $C d 45$ were all enriched in microglia, while the classical monocyte/macrophage marker $\mathrm{Ccr} 2$ was enriched in monocytes/macrophages isolated from RCAS tumors.

As further confirmation, we leveraged an independently-generated RNA sequencing dataset generated by the Joyce laboratory using the identical RCAS platform [5]. In this study, glioma-associated microglia were isolated from RCAS-induced gliomas based on $\mathrm{CD}_{4} 5^{+} \mathrm{CD} 11 \mathrm{~b}^{+} \mathrm{Ly}_{6 \mathrm{G}^{-}} \mathrm{Ly}_{6 \mathrm{C}^{-}} \mathrm{TdT}$ Tomato $^{+} \mathrm{GFP}^{-}$expression, whereas glioma-associated monocytes/macrophages were

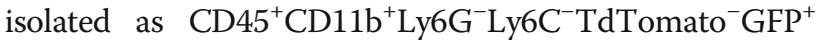

cells. Data were extracted, and the log2-fold change expression of glioma-associated microglia calculated relative to glioma-associated monocytes/macrophages (Fig. 5b). All eight SGmic genes were enriched in glioma-associated microglia in this RCAS dataset, with Slc2a5, Siglec-H, Gpr34 and P2ry12 exhibiting the highest expression, followed by Tmem119, Fcrls, P2ry13 and Olfml3, similar to the experimental data presented in Fig. 5a. The peripheral monocyte/macrophage marker genes $F 5, G d a, H p$, Sell and F10 were strongly enriched in glioma-associated monocytes/macrophages, followed by Emilin2. Mki67 expression did not show a significant difference between glioma-associated microglia and monocytes/macrophages, while $C 3$ was slightly enriched in the glioma-associated microglia fraction. Similarly, $C x 3 c r 1$ and $C d 11 b$ were only slightly enriched in glioma-associated microglia, while $\mathrm{Cd} 45$ and $\mathrm{Ccr} 2$ were enriched in glioma-associated monocytes/macrophages.

We then employed a second published RNA sequencing dataset derived from the GL261 glioma model system that was also analyzed by the Joyce laboratory [5]. GL261 glioblastoma tumors were implanted in wild-type C57BL/6J mice and glioma-associated microglia were isolated as $\mathrm{CD}_{4} 5^{+} \mathrm{CD} 11 \mathrm{~b}^{+} \mathrm{Ly} 6 \mathrm{G}^{-} \mathrm{Ly}_{6 \mathrm{C}^{-}} \mathrm{TdTomato}^{+}$cells by FACS sorting, while glioma-associated monocytes/ macrophages were isolated as $\mathrm{CD}_{4} 5^{+} \mathrm{CD} 11 \mathrm{~b}^{+}$Ly6$\mathrm{G}^{-}$Ly6C $^{-}$TdTomato $^{-}$cells. As above, we calculated $\log 2$-fold changes in gene expression (Fig. $5 \mathrm{c}$ ), and found that all eight identified microglia and peripheral monocyte/macrophage markers were significantly enriched in the glioma-associated microglia and monocyte/macrophage populations, respectively. Similar to the RCAS-TVA tumors, Slc2a 5 and Siglec- $H$ were expressed at the highest levels in GL261 glioma-associated microglia, followed by P2ry12 and Gpr34, while F10, Emilin2, $\mathrm{Gda}$, Sell and $H p$ showed the highest expression in glioma-associated monocytes/macrophages. As previously seen, C3 and Mki67 were only barely enriched in glioma-associated monocytes/macrophages. While $C d 11 b$ was enriched in GL261-derived monocytes/macrophages, the other classical monocyte marker genes (Cx3cr1, Cd45 and Ccr2) showed enrichment in GL261-derived microglia.

In addition, we assessed whether and how the pathologic condition of glioma affects SGmic gene expression in microglia by calculating the $\log 2$ fold changes of SGmic gene expression between glioma-associated microglia and healthy microglia in both glioma models and for all three RNA sequencing datasets as shown in Additional file 4: Figure S4. All SGmic genes showed a decrease in their expression levels in glioma-associated microglia as compared to healthy microglia across glioma models and datasets (log2-fold changes $=0.05-5.0$ ), except for Fcrls in the RCAS dataset published by 


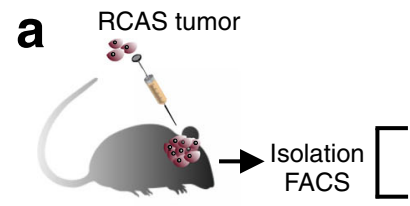

Ntv-a;Ink4a-Arf-/-Gli-luc

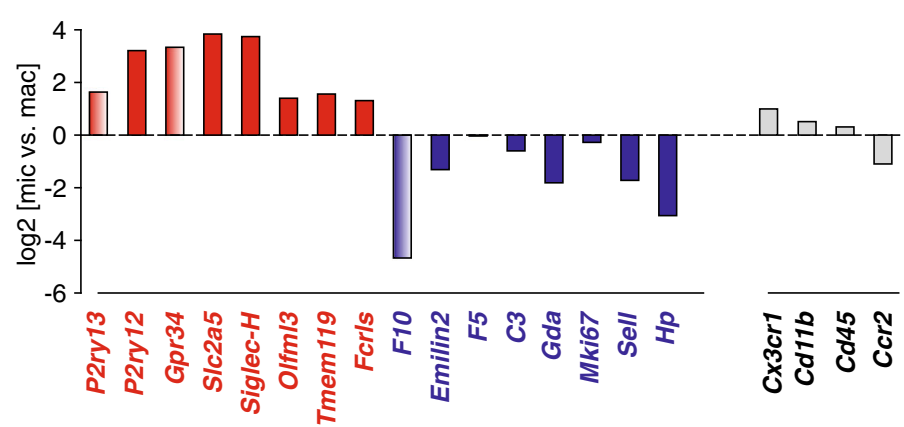

b

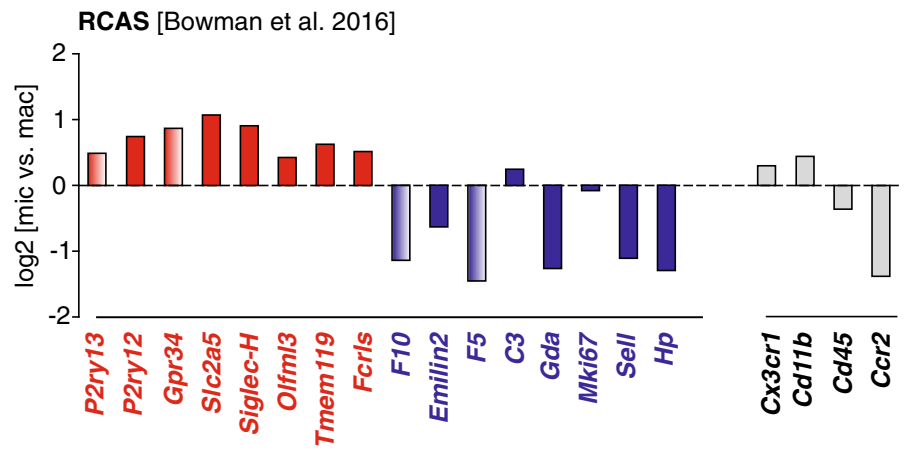

C

GL261 [Bowman et al. 2016]

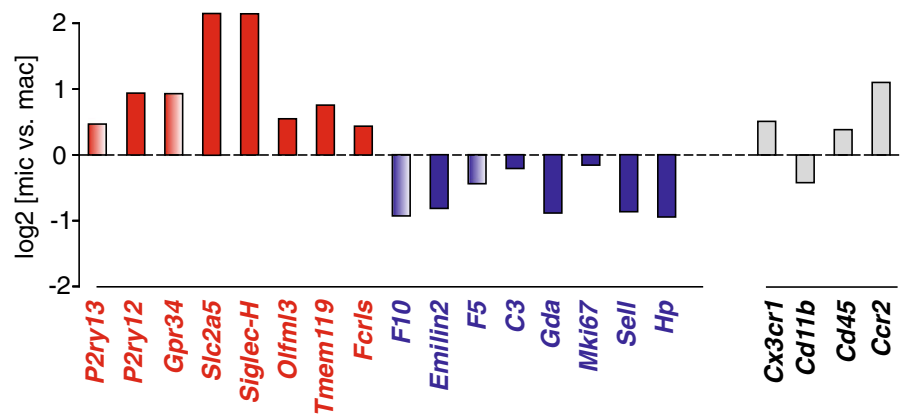

Fig. 5 Expression of SGmic and SGmac genes in glioma-associated microglia and monocytes/macrophages isolated from two different experimental mouse glioma models. (a) RCAS tumors were generated by injection of RCAS-PDFGB into Ntv-a;/nk4a-Arf-/-;Gli-luc mice, and after 5 weeks, gliomaassociated microglia were isolated as $\mathrm{CD} 11 \mathrm{~b}^{+}, \mathrm{CD} 45^{\text {low, }} \mathrm{F} 11 \mathrm{r}^{+}, \mathrm{Ly}_{6 \mathrm{G}^{\text {neg }}}$, Sell ${ }^{\text {neg }}, \mathrm{CD} 3^{\text {neg }}, \mathrm{CD} 19^{\text {neg }}, \mathrm{NK1} .1^{\text {neg }}$ cells, while glioma-associated monocytes/

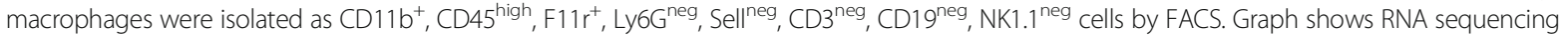
results of the two populations as $\log _{2}$-fold change expression of glioma-associated microglia to monocytes/macrophages for SGmic (P2ry 13, P2ry 12, Gpr34, Slc2a5, Siglec-H, Olfm/3, Tmem119, Fcrls) and SGmac (F10, Emilin, F5, C3, Gda, Mki67, Sell and Hp) genes, as well as the reference genes (CX3cr1, CD11b, CD45, Ccr2). Genes not detected in the previous proteomic analysis (see Fig. 4; SGmic: P2ry13, Gpr34 and SGmac: F10, F5) are colored with gradients. Graphs show $\log _{2}$-fold change expression of the SGmic and SGmac genes in glioma-associated microglia versus monocytes/macrophages isolated from (b) RCAS and (c) GL261 tumors derived from published sequencing data [5]. Glioma-associated microglia were isolated from RCAS

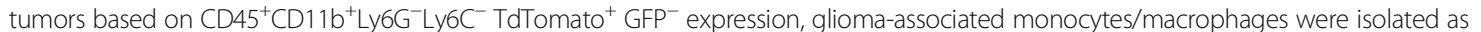

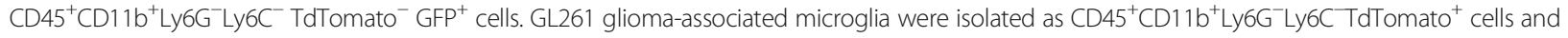
glioma-associated monocytes/macrophages were isolated as $\mathrm{CD}_{4} 5^{+} \mathrm{CD} 11 \mathrm{~b}^{+} \mathrm{Ly}_{6 \mathrm{G}}{ }^{-} \mathrm{Ly}_{6 \mathrm{C}}{ }^{-}$TdTomato ${ }^{-}$cells 
Bowman et al. [5]. Here, Slc2a5 and P2ry12 showed the strongest decrease across datasets, while Olfml3 and Tmem119 expression was least affected $(S l c 2 a 5=-0.68$ to $-5.0 ;$ P2ry $12=-0.61$ to -1.89 ; Olfml3 $=-0.05$ to 1.17; Tmem $119=-0.50$ to -1.99 ).

\section{The value of the SGmic and SGmac markers for understanding CNS pathogenesis}

Myeloid cells are highly dynamic cells whose transcriptomes are highly influenced by specific disease states, limiting their utility as reliable and stable cell identity markers. As such, microglia harbor gene expression patterns that reflect specific neuropathological conditions $[21,23]$. For example, in experimental mouse models of Alzheimer's disease (AD) and amyotrophic lateral sclerosis, unique microglia gene expression patterns have been reported [11, 27, 29]. Moreover, even within the same disease state (e.g., AD), microglia change their transcriptomes during the evolution of the pathologic process, reflecting the trajectory of cellular reprogramming in response to neurodegeneration and other CNS pathologies [31]. These temporal and spatial changes in microglial gene expression, and likely function, in the setting of CNS disease support the need for discriminatory markers that distinguish resident microglia from infiltrating monocyte/macrophage populations, so that the relative contributions of each monocyte population can be studied in greater detail.

The commonly used markers for distinguishing microglia from infiltrated monocytes/macrophages in the mouse system, including CD45, CX3CR1, and CCR2, have limitations that reflect their relative expression levels, which are presumed not to vary as a function of cellular context. In this regard, CD45 expression is frequently employed to distinguish microglia from peripheral monocytes/macrophages in FACS-based monocyte cell separations. However, this distinction relies upon gating the cells for differential expression levels, where microglia express low to intermediate levels and blood-derived monocytes/macrophages express high levels [15]. Obtaining clean separations is therefore dependent on the overlap between the different CD45-expressing populations, and does not consider that these levels could vary under pathological conditions. Germane to this latter issue, glioma-associated microglia increase CD45 expression in vivo, rendering them indistinguishable from $\mathrm{CD} 45^{\text {high }}$-expressing monocytes/macrophages [32]. In addition, we found that $C d 45$ expression was enriched in glioma-associated microglia relative to glioma-associated monocytes/macrophages (Fig. 5a, c). Similarly, while Cx3cr1 is often considered to be microglia-specific, circulating monocytes and resident tissue macrophages can also express Cx3cr1. Additionally, Ccr2, a blood-derived macrophage marker $[16-18,25]$, can be induced in microglia following lipopolysaccharide (LPS) treatment or reduced in blood-derived monocytes/ macrophages once they enter the brain in the context of CNS pathology $[1,4,11,40,47]$. This problem is further underscored by the observation that $\mathrm{Ccr} 2$ was enriched in glioma-associated monocytes/macrophages in both RCAS-tumor datasets, while it was enriched in gliomaassociated microglia isolated from the GL261-tumors (Fig. 5). Finally, we have previously shown that peripheral monocytes/macrophages acquire expression of a microglia-specific gene (F11r) upon entry into the brain using an experimental model of graft versus host disease and, rendering infiltrating monocytes/macrophages indistinguishable from resident microglia [33].

Similarly, several studies postulated novel and exclusive markers for identifying microglia in disorders affecting the CNS, including Tmem119 [3] and P2ry12 [7]. As such, TGF-ßR1, Fcrls, Gpr34, Sall1 and P2ry12 [7], as well as Siglec- $H$ [28], have been reported to be expressed at higher levels in microglia than in peripheral monocytes/macrophages. CD49D/Itga4 has also been described as a specific marker for bone-marrow derived macrophages due to its transcriptional suppression in microglia, and has been shown to separate the two cell populations in murine and human tumors [5]. In addition, TREM2 has similarly been suggested to distinguish infiltrated monocytes/macrophages from microglia [14]. However, none of these markers has been accepted as a universal standard.

The lack of a common set of markers to distinguish microglia from peripheral monocytes/macrophages that infiltrate the CNS has limited our understanding of the relative contributions of each of these monocyte populations to neurologic disease pathogenesis. In the present study, we employed an unbiased and comprehensive meta-analytic approach, combined with numerous experimental validations to identify two sets of highly reliable markers for microglia (SGmic) and peripheral monocytes/macrophages (SGmac). These SGmic and SGmac gene sets were then leveraged to separate microglia from infiltrating monocytes/macrophages in two different experimental mouse models of high-grade glioma. Within these marker sets, P2ry12, Tmem119, Slc2a5 and Fcrls performed best to discriminate microglia from other cell types, while Emilin2, Gda, Hp and Sell were the best markers for peripheral monocytes/macrophages. Throughout all investigated conditions and approaches, these markers were more reliable and performed better than the commonly used microglia/macrophage discriminators, underscoring their utility for discriminating these myeloid cell populations in both health and glioma and arguing for their use in future studies. Despite the observation that SGmic gene expression changed in glioma-associated microglia, and that LPS exposure 
decreased the expression of P2ry12, Tmem119, Fcrls and Olfml3 [3], the SGmic genes still outperformed the commonly used discriminators.

Based on the ability of our prime candidate microglia signature genes (P2ry12, Slc2a5, Tmem119 and Fcrls), as well as our top candidate marker genes for peripheral monocytes/macrophages (Gda and Hp, Sell and Emilin2), to stably distinguish these two populations in the normal brain, and in the context of high-grade glioma, it is interesting to note that a preliminary analysis indicates that P2ry12, Slc2a5 and Tmem119 genes are expressed in glioma-associated microglia isolated from a murine low-grade glioma model [41]. Thus, besides further proving the validity of SGmic and SGmac genes as reliable markers used in the field of glioma research, their applicability might also be explored in the broader context of other CNS diseases.

While Tmem119 and P2ry12 have already been shown to reliably identify human healthy microglia $[3,7]$, our results suggest that the other SGmic genes (P2ry13, Gpr34, Slc2a5, Siglec-H, Olfml3, Fcrls) may also serve as human microglia markers. Moreover, future studies might explore whether Tmem119, P2ry12 (and potentially other SGmic genes) might possess the ability to distinguish gliomaassociated microglia from glioma-associated monocytes/ macrophages in human glioma tissue.

Since the SGmic genes (P2ry12, Slc2a5, Tmem119 and Fcrls) and SGmac genes (Gda and Hp, Sell and Emilin2) were validated at the protein level and are predicted to be expressed at the plasma membrane, it becomes possible to consider them for future protein-based applications, such as Western blotting, immunocytochemistry, FACS analysis, and potentially for generating new mouse reporter or Cre driver lines.

\section{Conclusions}

Using large meta-analytic approach, we identified a robust panel of microglia and peripheral monocyte/macrophage markers, which were independently validated at the RNA and protein levels. The value of these discriminating marker sets was further explored in the setting of glioma, where they distinguished glioma-associated microglia from macrophages in two mouse glioblastoma models. Future studies employing these discriminatory genes/proteins to separate monocyte populations may facilitate the discovery of novel and distinct functions for microglia and infiltrating monocytes/macrophages in CNS disease.

\section{Additional files}

Additional file 1: Figure S1.. Expression levels of selected differentially expressed macrophage marker genes after hierarchical clustering in peripheral monocyte/macrophage subpopulations isolated from blood, spleen, peritoneum and bone marrow. (a) The differentially-expressed
SGmac genes, which were identified in cluster 1 (Cd24, Mki67, Gda, Anxa2 C3, Fn1, Slpi, Emilin2, F10) following hierarchical clustering of the 145 significantly enriched and specific peripheral monocyte/macrophage genes shared across all five datasets, are shown. Expression is shown as the log 2 fold change of expression of the peripheral monocyte/ macrophage subpopulations isolated from blood (dark green; [5]), spleen (light green; [7]), peritoneum (light blue; [22]) and bone marrow (dark grey; [33]) compared to microglia for each of the datasets. For bone marrow-derived monocyte/macrophages, the RNA-sequencing dataset from Pong et al. is shown [33]. (b) The differentially-expressed SGmac genes, which were identified in cluster 2 (Hp, Sell, Mgst1 and S100a6) following hierarchical clustering of the 145 significantly enriched and specific peripheral monocyte/macrophage genes shared across all five datasets, are shown. Expression is shown as the $\log 2$ fold change of expression of the peripheral monocyte/macrophage subpopulations isolated from blood (dark green; [5]), spleen (light green; [7]), peritoneum (light blue; [22]) and bone marrow (dark grey; [33]) compared to microglia for each of the datasets. For bone marrow- derived monocyte/macrophages, the RNA-sequencing dataset from Pong et al. is shown [33]. (PDF $393 \mathrm{~kb})$

Additional file 2: Figure S2. Spatial visualization, clustering and expression of SGmic, SGmac and classical monocyte/macrophage marker genes in single cell sequencing data derived from brain myeloid and bone marrow cells. (a) t-distributed Stochastic Neighbor Embedding ( $t$-SNE) spatial visualization and clustering of brain myeloid single cells (microglia; turquoise) dataset and bone marrow single cells (red) dataset derived from the single cell sequencing data of the Tabula Muris Consortium [42]. The right panel depicts clusters 1-16 represent all different cell populations detected by automatic clustering (Seurat FindCluster function). (b) t-SNEs showing the expression of the SGmic genes within the spatial distribution of brain myeloid and bone marrow cells. Identified SGmic genes from the analyzed single cell sequencing dataset comprise P2ry13, P2ry12, Gpr34, S/c2a5, Olfm/3, Tmem 119. (c) t-SNEs showing the expression of the SGmac genes (F10, Emilin2, F5, C3, Gda, Mki67, Sell, Hp) within the spatial distribution of brain myeloid and bone marrow cells. (d) t-SNEs showing the expression of the canonical monocyte/macrophage markers $(\mathrm{C} \times 3 \mathrm{Cr} 1, \mathrm{Cd} 11 \mathrm{~b}, \mathrm{Cd} 45$ and $\mathrm{C} \mathrm{Cr} 2)$ within the spatial distribution of brain myeloid and bone marrow cells. (PDF $14645 \mathrm{~kb}$ )

Additional file 3: Figure S3. Validation of SGmic and SGmac genes in microglia and peripheral monocytes/macrophages freshly isolated from two different mouse models. (a) Microglia (CD11 b ${ }^{+}$CD45 low; red) and circulating spleen monocytes (CD11 b $\mathrm{b}^{+}$CD $45^{\text {high }}$ Ly6 $\mathrm{G}^{\text {low }}$ Ly6 $\mathrm{C}^{\text {high }}$; blue) were freshly isolated from 12-week-old male C57/Bl6 WT mice by FACS and the expression of SGmic genes (P2ry13, P2ry12, Gpr34, Slc2a5, Siglech, Olfm/3, Tmem 119, and Fcrls; red) and SGmac genes (F10, Emilin2, F5, C3, Gda, Mki67, Sell, Hp; blue) determined by quantitative RT-PCR. Bar graphs represent the fold change expression of marker genes normalized to Hprt, where SGmic genes (red) are shown in relation to the spleen monocyte/

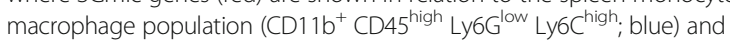
SGmac genes (blue) compared to microglia $(n=3)$. For statistical analysis, unpaired t-tests were performed. ${ }^{*}=P<0.05 ;{ }^{* *}=P<0.01 ;{ }^{* * *}=P<0.001$. (b) Microglia and spleen monocytes/macrophages were freshly isolated from 8 to 12 -week-old male $C \times 3 \mathrm{Cr} 7^{\text {GFPMT }} ; C \mathrm{Cr} 2^{\mathrm{RPPNT}}$ mice by FACS, GFP $^{+}$RFP $^{-}$cells representing microglia (red), RFP ${ }^{+} \mathrm{GFP}^{+}$(blue) and RFP+ GFP(purple) cells representing spleen monocytes/macrophages. The expression of SGmic genes (P2ry13, P2ry12, Gpr34, Slc2a5, Siglech, Olfm/3, Tmem119, and Fcrls; red) and SGmac genes (F10, Emilin2, F5, C3, Gda, Mki67, Sell, Hp; blue) was determined by quantitative RT-PCR. Bar graphs represent the fold change expression of each gene normalized to Hprt, where SGmic genes (red) are shown in relation to spleen monocytes/macrophages (RFP $\mathrm{GFP}^{+}$; blue) and SGmac genes compared to microglia (GFP'RFP- cells; $n=3$ ). For statistical analysis, one-way ANOVA following Bonferroni's multiple comparison test was performed. ${ }^{*}=P<0.05 ;{ }^{* *}=P<0.01 ;{ }^{* *}=P<0.001$. (PDF $401 \mathrm{~kb}$ )

Additional file 4: Figure S4. Expression of SGmic genes in gliomaassociated microglia as compared to healthy microglia in RNA sequencing datasets derived from healthy, RCAS glioma or GL261 glioma mice. (a) The log-fold change expression of SGmic genes (P2ry13, P2ry12, Gpr34, Slc2a5, Siglec-H, Olfm/3, Tmem119, Fcrls) in glioma-associated 
microglia isolated from experimental RCAS tumors compared to microglia isolated from healthy control brains is shown. Expression data were extracted from RNA sequencing data generated by our group. (b) The log-fold change expression of SGmic genes (P2ry13, P2ry12, Gpr34, S/c2a5, Siglec-H, Olfm/3, Tmem119, Fcrls) in glioma-associated microglia isolated from RCAS tumors compared to microglia isolated from healthy control brains is shown. Expression data were extracted from published RNA sequencing data [5]. (c) Graph shows the log-fold change expression of SGmic genes (P2ry13, P2ry12, Gpr34, S/c2a5, Siglec-H, Olfm/3, Tmem119, Fcrls) in glioma-associated microglia isolated from GL261 tumors as compared to microglia isolated from healthy control brains. Expression data were extracted from published RNA sequencing data [5]. (PDF 394 kb)

\section{Acknowledgements}

We thank Regina Piske, Maren Wendt, Nadine Scharek, and Michaela SeegerZografakis for technical assistance. We thank the FACS facility and the proteomics facility of the Max Delbrück Center for Molecular Medicine in the Helmholtz Association, Berlin, Germany for technical assistance.

\section{Funding}

The work was supported by the NeuroCure Cluster of Excellence, a Berlin Institute of Health/Einstein fellowship grant to D.H.G. and H.K. D.H.G. is an Alexander von Humboldt Fellow.

\section{Authors' contributions}

VH performed cell isolation and qRT-PCR experiments; ROV, and DPH performed the RNA and protein bioinformatic analyses; WWP, ZC, DH, VM, AL, JW, and EM provided new RNA datasets; PM, MS, SS, HK, and DHG supervised the studies and conceived the project; VH, MS, HK and DHG wrote the manuscript. All authors read and approved the final manuscript.

\section{Ethics approval and consent to participate}

Animal experiments in Berlin were performed in strict accordance with the German Animal Protection Law as approved by the Regional Office for Health and Social Services in Berlin (Landesamt für Gesundheit und Soziales, Berlin, Germany, Permit Number (T0014/08, O360/09, A-0376/17). Adult mice were euthanized by intraperitoneal injection of pentobarbital (Narcoren, Merial GmbH, Hallbergmoos, Germany). All efforts were made to minimize pain and suffering.

Animals for the experimental glioma studies were housed in the Cleveland Clinic Biological Resource Unit or the Emory University Division of Animal Resources. All experimental procedures were approved by the Institutional Animal Care and Use Committee of the Cleveland Clinic (Animal Protocol 2013-1029; approved June 25, 2013) and Emory University (Protocol \#2003253; approved September 15, 2015), and performed in strict accordance with the recommendations in the Guide for the Care and Use of Laboratory Animals of the National Institutes of Health. All surgeries were performed under anesthesia, and all efforts were made to minimize suffering.

\section{Competing interests}

The authors declare that they have no competing interests.

\section{Publisher's Note}

Springer Nature remains neutral with regard to jurisdictional claims in published maps and institutional affiliations.

\section{Author details}

${ }^{1}$ Max Delbrück Center for Molecular Medicine in the Helmholtz Association, Berlin, Germany. ${ }^{2}$ Department of Neurology, Washington University School of Medicine, Box 8111, 660 S. Euclid Avenue, St. Louis, MO 63110, USA ${ }^{3}$ Department of Pediatrics, Emory University, Atlanta, GA, USA. ${ }^{4}$ McDonnell Genome Institute, Washington University School of Medicine, St. Louis, MO, USA.

Received: 17 December 2018 Accepted: 22 January 2019 Published online: 14 February 2019

\section{References}

1. Banisadr G, Quéraud-Lesaux F, Boutterin MC, Pélaprat D, Zalc B, Rostène W, Haour F, Parsadaniantz SM (2002) Distribution, cellular localization and functional role of CCR2 chemokine receptors in adult rat brain. J Neurochem 81:257-269. https://doi.org/10.1046/j.1471-4159.2002.00809.x

2. Baumgarth $N$, Roederer $M$ (2000) A practical approach to multicolor flow cytometry for immunophenotyping. J Immunol Methods 243:77-97. https:// doi.org/10.1016/S0022-1759(00)00229-5

3. Bennett ML, Bennett FC, Liddelow SA, Ajami B, Zamanian JL, Fernhoff NB, Mulinyawe SB, Bohlen CJ, Adil A, Tucker A, Weissman IL, Chang EF, Li G, Grant GA, Hayden Gephart MG, Barres BA (2016) New tools for studying microglia in the mouse and human CNS. Proc Natl Acad Sci 113:E1738E1746. https://doi.org/10.1073/pnas.1525528113

4. Boddeke EWGM, Meigel I, Frentzel S, Gourmala NG, Harrison JK, Buttini M, Spleiss O, Gebicke-Härter P (1999) Cultured rat microglia express functional -chemokine receptors. J Neuroimmunol 98:176-184. https://doi.org/10. 1016/S0165-5728(99)00096-X

5. Bowman RL, Klemm F, Akkari L, Pyonteck SM, Sevenich L, Quail DF, Dhara S, Simpson K, Gardner EE, lacobuzio-Donahue CA, Brennan CW, Tabar V, Gutin PH, Joyce JA (2016) Macrophage ontogeny underlies differences in tumorspecific education in brain malignancies. Cell Rep 17:2445-2459. https://doi. org/10.1016/j.celrep.2016.10.052

6. Brandenburg S, Müller A, Turkowski K, Radev YT, Rot S, Schmidt C, Bungert AD, Acker G, Schorr A, Hippe A, Miller K, Heppner FL, Homey B, Vajkoczy P (2016) Resident microglia rather than peripheral macrophages promote vascularization in brain tumors and are source of alternative pro-angiogenic factors. Acta Neuropathol 131:365-378. https://doi.org/10.1007/s00401-015-1529-6

7. Butovsky O, Jedrychowski MP, Moore CS, Cialic R, Lanser AJ, Gabriely G, Koeglsperger T, Dake B, Wu PM, Doykan CE, Fanek Z, Liu L, Chen Z, Rothstein JD, Ransohoff RM, Gygi SP, Antel JP, Weiner HL (2014) Identification of a unique TGF- $\beta$-dependent molecular and functional signature in microglia. Nat Neurosci 17:131-143. https://doi.org/10.1038/nn.3599

8. Cardona AE, Huang D, Sasse ME, Ransohoff RM (2006) Isolation of murine microglial cells for RNA analysis or flow cytometry. Nat Protoc 1:1947-1951. https://doi.org/10.1038/nprot.2006.327

9. Chen Z, Feng X, Herting CJ, Garcia VA, Nie K, Pong WW, Rasmussen R, Dwivedi B, Seby S, Wolf SA, Gutmann DH, Hambardzumyan D (2017) Cellular and molecular identity of tumor-associated macrophages in glioblastoma. Cancer Res 77:2266-2278. https://doi.org/10.1158/0008-5472.CAN-16-2310

10. Chiu IM, Morimoto ETA, Goodarzi H, Liao JT, Keeffe SO, Phatnani HP, Muratet M, Carroll MC, Levy S, Tavazoie S, Myers RM, Maniatis T (2014) NIH Public Access model. 4:385-401. doi: https://doi.org/10.1016/j.celrep.2013.06.018.A

11. Chiu IM, Morimoto ETA, Goodarzi H, Liao JT, O'Keeffe S, Phatnani HP, Muratet M, Carroll MC, Levy S, Tavazoie S, Myers RM, Maniatis T (2013) A neurodegeneration-specific gene-expression signature of acutely isolated microglia from an amyotrophic lateral sclerosis mouse model. Cell Rep 4: 385-401. https://doi.org/10.1016/j.celrep.2013.06.018

12. Cox J, Mann M (2008) MaxQuant enables high peptide identification rates, individualized p.p.b.-range mass accuracies and proteome-wide protein quantification. Nat Biotechnol 26:1367-1372. https://doi.org/10.1038/nbt.1511

13. de Vries HE, Kuiper J, de Boer AG, Van Berkel TJ, Breimer DD (1997) The Blood-Brain Barrier in Neuroinflammatory. Pharmacol Rev 49:143-155.

14. Fahrenhold M, Rakic S, Classey J, Brayne C, Ince PG, Nicoll JAR, Boche D (2017) TREM2 expression in the human brain: a marker of monocyte recruitment? Brain Pathol:595-602. https://doi.org/10.1111/bpa.12564

15. Ford AL, Goodsall AL, Hickey WF, Sedgwick JD (1995) Normal adult ramified microglia separated from other central nervous system macrophages by flow cytometric sorting: phenotypic differences defined and direct ex vivo antigen presentation to myelin basic protein-reactive CD4+ T cells compared. J Immunol 154:4309-4321. https://doi.org/10.4049/jimmunol.173.6.3916

16. Gautier EL, Shay T, Miller J, Greter M, Jakubzick C, Ivanov S, Helft J, Chow A, Elpek KG, Gordonov S, Mazloom AR, Ma'Ayan A, Chua WJ, Hansen TH, Turley SJ, Merad M, Randolph GJ, Best AJ, Knell J, Goldrath A, Brown B, Jojic V, Koller D, Cohen N, Brenner M, Regev A, Fletcher A, Bellemare-Pelletier A, Malhotra D, Jianu R, Laidlaw D, Collins J, Narayan K, Sylvia K, Kang J, Gazit R, Garrison BS, Rossi DJ, Kim F, Rao TN, Wagers A, Shinton SA, Hardy RR, Monach P, Bezman NA, Sun JC, Kim CC, Lanier LL, Heng T, Kreslavsky T, Painter M, Ericson J, Davis S, Mathis D, Benoist C (2012) Gene-expression profiles and transcriptional regulatory pathways that underlie the identity and diversity of mouse tissue macrophages. Nat Immunol 13:1118-1128. https://doi.org/10.1038/ni.2419

17. Geissmann F, Jung S, Littman DR (2003) Blood monocytes consist of two principal subsets with distinct migratory properties cytes that develop in the BM from dividing monoblasts, are released in the bloodstream as 
nondividing cells, and enter tissues, where their fate remains largely a. Immunity 19:71-82

18. Gomez Perdiguero E, Schulz C, Geissmann F (2013) Development and homeostasis of "resident" myeloid cells: the case of the microglia. Glia 61: 112-120. https://doi.org/10.1002/glia.22393

19. Govindan R, Ding L, Griffith M, Subramanian J, Dees ND, Kanchi KL, Maher CA, Fulton R, Fulton L, Wallis J, Chen K, Walker J, McDonald S, Bose R, Ornitz D, Xiong D, You M, Dooling DJ, Watson M, Mardis ER, Wilson RK (2012) Genomic landscape of non-small cell lung cancer in smokers and neversmokers. Cell 150:1121-1134. https://doi.org/10.1016/j.cell.2012.08.024

20. Hambardzumyan D, Amankulor NM, Helmy KY, Becher OJ, Holland EC (2009) Modeling adult gliomas using RCAS/t-va technology. Transl Oncol 2: 89-IN6. https://doi.org/10.1593/tlo.09100

21. Hammond TR, Dufort C, Dissing-Olesen L, Giera S, Young A, Wysoker A, Walker AJ, Gergits F, Segel M, Nemesh J, Marsh SE, Saunders A, Macosko E, Ginhoux F, Chen J, Franklin RJM, Piao X, McCarroll SA, Stevens B (2018) Single-cell RNA sequencing of microglia throughout the mouse lifespan and in the injured brain reveals complex cell-state changes. Immunity:1-19. https://doi.org/10.1016/j.immuni.2018.11.004

22. Hickman SE, Kingery ND, Ohsumi TK, Borowsky ML, Wang LC, Means TK, El Khoury J (2013) The microglial sensome revealed by direct RNA sequencing. Nat Neurosci 16:1896-1905. https://doi.org/10.1038/nn.3554

23. Hirbec H, Marmai C, Duroux-Richard I, Roubert C, Esclangon A, Croze S, Lachuer J, Peyroutou R, Rassendren F (2018) The microglial reaction signature revealed by RNAseq from individual mice. Glia 66:971-986. https://doi.org/10.1002/glia.23295

24. Imai T, Hieshima K, Haskell C, Baba M, Nagira M, Nishimura M, Kakizaki M, Takagi S, Nomiyama H, Schall TJ, Yoshie O (1997) Identification and molecular characterization of fractalkine receptor CX3CR1, which mediates both leukocyte migration and adhesion. Cell 91:521-530. https://doi.org/10. 1016/S0092-8674(00)80438-9

25. Jung S, Aliberti J, Graemmel P, Sunshine MJ, Kreutzberg GW, Sher A, Littman DR (2000) Analysis of Fractalkine receptor CX3CR1 function by targeted deletion and green fluorescent protein reporter gene insertion. Mol Cell Biol 20:4106-4114. https://doi.org/10.1128/MCB.20.11.4106-4114.2000

26. Kanashova T, Popp O, Orasche J, Karg E, Harndorf H, Stengel B, Sklorz M, Streibel T, Zimmermann R, Dittmar G (2015) Differential proteomic analysis of mouse macrophages exposed to adsorbate-loaded heavy fuel oil derived combustion particles using an automated sample-preparation workflow aerosols and health. Anal Bioanal Chem 407:5965-5976. https://doi.org/10. 1007/s00216-015-8595-4

27. Keren-Shaul H, Spinrad A, Weiner A, Matcovitch-Natan O, Dvir-Szternfeld R, Ulland TK, David E, Baruch K, Lara-Astaiso D, Toth B, Itzkovitz S, Colonna M, Schwartz M, Amit I (2017) A unique microglia type associated with restricting development of Alzheimer's disease. Cell 169:1276-1290.e17. https://doi.org/10.1016/j.cell.2017.05.018

28. Konishi H, Kobayashi M, Kunisawa T, Imai K, Sayo A, Malissen B, Crocker PR, Sato K, Kiyama H (2017) Siglec-H is a microglia-specific marker that discriminates microglia from CNS-associated macrophages and CNS infiltrating monocytes. Glia 65:1927-1943. https://doi.org/10.1002/glia.23204

29. Litvinchuk A, Wan Y-W, Swartzlander DB, Chen F, Cole A, Propson NE, Wang Q, Zhang B, Liu Z, Zheng H (2018) Complement C3aR inactivation attenuates tau pathology and reverses an immune network deregulated in Tauopathy models and Alzheimer's disease. Neuron:1-17. https://doi.org/10. 1016/j.neuron.2018.10.031

30. Mardis ER, Ding L, Dooling DJ, Larson DE, McLellan MD, Chen K, Koboldt DC, Fulton RS, Delehaunty KD, McGrath SD, Fulton LA, Locke DP, Magrini VJ, Abbott RM, Vickery TL, Reed JS, Robinson JS, Wylie T, Smith SM, Carmichael L, Eldred JM, Harris CC, Walker J, Peck JB, Du F, Dukes AF, Sanderson GE, Brummett AM, Clark E, McMichael JF, Meyer RJ, Schindler JK, Pohl CS, Wallis JW, Shi X, Lin L, Schmidt H, Tang Y, Haipek C, Wiechert ME, Ivy JV, Kalicki J, Elliott G, Ries RE, Payton JE, Westervelt P, Tomasson MH, Watson MA, Baty J, Heath S, Shannon WD, Nagarajan R, Link DC, Walter MJ, Graubert TA, DiPersio JF, Wilson RK, Ley TJ (2009) Recurring mutations found by sequencing an acute myeloid leukemia genome. N Engl J Med 361:10581066. https://doi.org/10.1056/NEJMoa0903840

31. Mathys H, Adaikkan C, Gao F, Young JZ, Hemberg M, Jager PL De, Ransohoff RM, Regev A (2017) at Single-Cell Resolution. 21:366-380. doi: https://doi.org/10.1016/j.celrep.2017.09.039.Temporal
32. Müller A, Brandenburg S, Turkowski K, Müller S, Vajkoczy P (2015) Resident microglia, and not peripheral macrophages, are the main source of brain tumor mononuclear cells. Int J Cancer 137:278-288. https://doi.org/10.1002/ijc.29379

33. Pong WW, Walker J, Wylie T, Magrini V, Luo J, Emnett RJ, Choi J, Cooper ML, Griffith M, Griffith OL, Rubin JB, Fuller GN, Piwnica-Worms D, Feng X, Hambardzumyan D, DiPersio JF, Mardis ER, Gutmann DH (2013) F11R is a novel monocyte prognostic biomarker for malignant glioma. PLoS One 8:113. https://doi.org/10.1371/journal.pone.0077571

34. Prinz M, Erny D, Hagemeyer N (2017) Ontogeny and homeostasis of CNS myeloid cells. Nat Immunol 18:385-392. https://doi.org/10.1038/ni.3703

35. Rappsilber J, Mann M, Ishihama Y (2007) Protocol for micro-purification, enrichment, pre-fractionation and storage of peptides for proteomics using StageTips. Nat Protoc 2:1896-1906. https://doi.org/10.1038/nprot.2007.261

36. Roberts A, Pimentel $H$, Trapnell C, Pachter $L$ (2011) Identification of novel transcripts in annotated genomes using RNA-seq. Bioinformatics 27:23252329. https://doi.org/10.1093/bioinformatics/btr355

37. Roberts A, Trapnell C, Donaghey J, Rinn JL, Pachter L (2011) Improving RNASeq expression estimates by correcting for fragment bias. Genome Biol 12: R22. https://doi.org/10.1186/gb-2011-12-3-r22

38. Seligman AM, Shear M (1939) Experimental production of brain tumors in mice with methylcholanthrene. Cancer Res 37:364-395.

39. Shevchenko A, Tomas H, Havliš J, Olsen JV, Mann M (2007) In-gel digestion for mass spectrometric characterization of proteins and proteomes. Nat Protoc 1:2856-2860. https://doi.org/10.1038/nprot.2006.468

40. Sivakumar V, Foulds WS, Luu CD, Ling E, Kaur C (2011) Retinal ganglion cell death is induced by microglia derived pro-inflammatory cytokines in the hypoxic neonatal retina. J Pathol:245-260. https://doi.org/10.1002/path.2858

41. Solga AC, Pong WW, Kim KY, Cimino PJ, Toonen JA, Walker J, Wylie T, Magrini V, Griffith M, Griffith OL, Ly A, Ellisman MH, Mardis ER, Gutmann DH (2015) RNA sequencing of tumor-associated microglia reveals Ccl5 as a stromal chemokine critical for Neurofibromatosis-1 glioma growth. Neoplasia 17:776-788. https://doi.org/10.1016/j.neo.2015.10.002

42. Tabula T, Consortium M (2018) Single-cell transcriptomics of 20 mouse organs creates a Tabula Muris. Nature. https://doi.org/10.1038/s41586-0180590-4

43. Trapnell C, Hendrickson DG, Sauvageau M, Goff L, Rinn JL, Pachter L (2013) Differential analysis of gene regulation at transcript resolution with RNA-seq Nat Biotechnol 31:46-53. https://doi.org/10.1038/nbt.2450

44. Trapnell C, Pachter L, Salzberg SL (2009) TopHat: discovering splice junctions with RNA-Seq. Bioinformatics 25:1105-1111. https://doi.org/10. 1093/bioinformatics/btp120

45. Wolf SA, Boddeke HWGM, Kettenmann H (2017) Microglia in physiology and disease. Annu Rev Physiol 79:619-643. https://doi.org/10.1146/annurevphysiol-022516-034406

46. Yona S, Kim KW, Wolf Y, Mildner A, Varol D, Breker M, Strauss-Ayali D, Viukov S, Guilliams M, Misharin A, Hume DA, Perlman H, Malissen B, Zelzer E, Jung $S$ (2013) Fate mapping reveals origins and dynamics of monocytes and tissue macrophages under homeostasis. Immunity 38:79-91. https://doi.org/ 10.1016/j.immuni.2012.12.001

47. Zhang J, Shi XQ, Echeverry S, Mogil JS, De Koninck Y, Rivest S (2007) Expression of CCR2 in both resident and bone marrow-derived microglia plays a critical role in neuropathic pain. J Neurosci 27:12396-12406. https:// doi.org/10.1523/JNEUROSCI.3016-07.2007

48. Zhang Y, Chen K, Sloan SA, Bennett ML, Scholze AR, O'Keeffe S, Phatnani HP, Guarnieri P, Caneda C, Ruderisch N, Deng S, Liddelow SA, Zhang C, Daneman R, Maniatis T, Barres BA, Wu JQ (2014) An RNA-sequencing transcriptome and splicing database of glia, neurons, and vascular cells of the cerebral cortex. J Neurosci 34:11929-11947. https://doi.org/10.1523/ JNEUROSCI.1860-14.2014 